This article has been accepted for publication in Monthly Notices of the Royal Astronomical Society. (0: 2018 The Authors. Published by Oxford University Press on behalf of the Royal Astronomical Society. All rights reserved. 


\title{
The Chandra COSMOS Legacy Survey: Compton thick AGN at high redshift
}

\author{
G. Lanzuisi, ${ }^{1,2}$ F. Civano, ${ }^{3,4 \star ~ S . ~ M a r c h e s i, ~}{ }^{5}$ A. Comastri, ${ }^{2}$ M. Brusa, ${ }^{1,2}$ R. Gilli, ${ }^{2}$ \\ C. Vignali, ${ }^{1,2}$ G. Zamorani, ${ }^{2}$ M. Brightman, ${ }^{6}$ R. E. Griffiths ${ }^{7}$ and A. M. Koekemoer ${ }^{8}$ \\ ${ }^{1}$ Dipartimento di Fisica e Astronomia, Università di Bologna, Via Gobetti 93/2, I-40129 Bologna, Italy \\ ${ }^{2}$ INAF - Osservatorio di Astrofisica e Scienza dello Spazio di Bologna, Via Gobetti 93/3, I-40129 Bologna, Italy \\ ${ }^{3}$ Harvard-Smithsonian Center for Astrophysics, 60 Garden Street, Cambridge, MA 02138, USA \\ ${ }^{4}$ Yale Center for Astronomy and Astrophysics, 260 Whitney Avenue, New Haven, CT 06520, USA \\ ${ }^{5}$ Department of Physics and Astronomy, Clemson University, Clemson, SC 29634, USA \\ ${ }^{6}$ Cahill Center for Astrophysics, California Institute of Technology, 1216 East California Boulevard, Pasadena, CA 91125, USA \\ ${ }^{7}$ Department of Physics and Astronomy, University of Hawaii at Hilo, 200 W. Kawili Street, Hilo, HI 96720, USA \\ ${ }^{8}$ Space Telescope Science Institute, 3700 San Martin Drive, Baltimore, MD 21218, USA
}

Accepted 2018 July 25. Received 2018 July 25; in original form 2018 March 22

\begin{abstract}
The existence of a large population of Compton thick (CT; $N_{\mathrm{H}}>10^{24} \mathrm{~cm}^{-2}$ ) active galactic nuclei (AGN) is a key ingredient of most cosmic X-ray background synthesis models. However, direct identification of these sources, especially at high redshift, is difficult due to flux suppression and complex spectral shape produced by CT obscuration. We explored the Chandra Cosmological Evolution Survey (COSMOS) Legacy point source catalogue, comprising 1855 sources to select, via X-ray spectroscopy, a large sample of CT candidates at high redshift. Adopting a physical model to reproduce the toroidal absorber and a Monte-Carlo sampling method, we selected 67 individual sources with $>5$ per cent probability of being CT, in the redshift range $0.04 \lesssim z \lesssim 3.5$. The sum of the probabilities above $N_{\mathrm{H}}>10^{24} \mathrm{~cm}^{-2}$ gives a total of 41.9 effective $\mathrm{CT}$, corrected for classification bias. We derive number counts in the $2-10 \mathrm{keV}$ band in three redshift bins. The observed $\log N-\log S$ is consistent with an increase of the intrinsic $\mathrm{CT}$ fraction $\left(f_{\mathrm{CT}}\right)$ from $\sim 0.30$ to $\sim 0.55$ from low to high redshift. When rescaled to a common luminosity $\left(\log \left(L_{X} / \mathrm{erg} \mathrm{s}^{-1}\right)=44.5\right)$, we find an increase from $f_{\mathrm{CT}}=0.19_{-0.06}^{+0.07}$ to $0.30_{-0.08}^{+0.10}$ and $f_{\mathrm{CT}}=0.49_{-0.11}^{+0.12}$ from low to high $z$. This evolution can be parametrized as $f_{\mathrm{CT}}=0.11_{-0.04}^{+0.05}(1+z)^{1.11 \pm 0.13}$. Thanks to Hubble Space Telescope-Advanced Camera for Surveys deep imaging, we find that the fraction of CT AGN in mergers/interacting systems increases with luminosity and redshift and is significantly higher than for non-CT AGN hosts.
\end{abstract}

Key words: galaxies: active-galaxies: nuclei-X-rays: galaxies.

\section{INTRODUCTION}

We have known for 20 yr that a large fraction (up to 50 per cent) of local active galactic nuclei (AGN) are obscured by large amounts of gas and dust (e.g. Risaliti, Maiolino \& Salvati 1999), above the Compton thick (CT) threshold. ${ }^{1}$ A sizable intrinsic fraction of CT $\operatorname{AGN}\left(f_{\mathrm{CT}}\right)$ is required in most cosmic $\mathrm{X}$-ray background (CXB) synthesis models (e.g. Comastri et al. 1995; Gilli, Comastri \& Hasinger

\footnotetext{
^E-mail: giorgio.lanzuisi2@unibo.it

${ }^{1}$ Equivalent hydrogen column density $N_{\mathrm{H}} \geq \sigma_{\mathrm{T}}^{-1} \sim 1.6 \times 10^{24} \mathrm{~cm}^{-2}$. At these high column densities the obscuration is mainly due to Compton scattering, rather than photoelectric absorption.
}

2007, hereafter G07) in order to reproduce the hump observed at 20-30 keV in the background spectrum (e.g. Ballantyne et al. 2011). However, the value of $f_{\mathrm{CT}}$ derived in this way is highly uncertain, ranging from $\sim 0.1$ to $\sim 0.3-0.4$ (G07; Treister, Urry \& Virani 2009; Ueda et al. 2014) due to degeneracies between several model parameters, e.g. the primary continuum photon index, the reflection fraction, the $N_{\mathrm{H}}$ distribution above $10^{24} \mathrm{~cm}^{-2}$, and the high energy cut-off (see e.g. Akylas et al. 2012).

$\mathrm{X}$-rays are able to provide the smoking gun of CT obscuration, thanks to the unique spectral signatures observable, i.e. the flat continuum below $\sim 10 \mathrm{keV}$ and the strong $\mathrm{Fe} \mathrm{K} \alpha$ emission line at $6.4 \mathrm{keV}$. Furthermore, above $L_{\mathrm{X}} \sim 10^{42} \mathrm{erg} \mathrm{s}^{-1}$ in the $2-10 \mathrm{keV}$ band, the contamination by star-forming galaxies is almost negligible. Fi- 
nally, X-ray spectroscopy is favoured by the redshift effect: going at high redshift, the Compton hump at 20-30 keV becomes observable by Chandra and XMM-Newton, and the Fe $\mathrm{K} \alpha$ line moves toward lower energies, where the effective area of current X-ray telescopes is larger.

However, collecting large samples of CT AGN beyond the local Universe remains difficult for three main reasons.

(i) The observed fraction of CT AGN steeply rises from $\sim 0$ to the intrinsic value (e.g. 0.3-0.4) only below a certain flux (e.g. $F \ll$ $10^{-14} \mathrm{erg} \mathrm{cm}^{-2} \mathrm{~s}^{-1}$ in the $2-10 \mathrm{keV}$ band, or $10^{-13} \mathrm{erg} \mathrm{cm}^{-2} \mathrm{~s}^{-1}$ in the 10-40 keV band; see e.g. G07; Ricci et al. 2015) and therefore it is mandatory to reach deep sensitivities over large areas in order to collect sizable samples of CT AGN.

(ii) For a given intrinsic luminosity, CT AGN are a factor of 30-50 fainter, below $10 \mathrm{keV}$ rest frame, than unobscured AGN, requiring long exposures to collect even a few tens of X-ray counts per source.

(iii) The transition between Compton-thin (C-thin) and CT absorption (i.e. below or above $N_{\mathrm{H}}>\sigma_{\mathrm{T}}^{-1} \sim 1.6 \times 10^{24} \mathrm{~cm}^{-2}$ ) is smooth (Murphy \& Yaqoob 2009, hereafter MY09), requiring a tailored analysis (see e.g. Buchner et al. 2014) with the use of the full $N_{\mathrm{H}}$ probability distribution function (PDF) when selecting CT AGN in order to avoid misclassification in one direction or the other.

For these reasons, even in the deepest X-ray fields, different analysis of the same samples (e.g. Tozzi et al. 2006; Brightman \& Ueda 2012, hereafter BU12; Georgantopoulos et al. 2013) give results not always in agreement (see Castelló-Mor et al. 2013; Liu et al. 2017).

NUSTAR, sensitive above $10 \mathrm{keV}$, is now placing new constraints on the observed $f_{\mathrm{CT}}$ at low redshift and relatively bright fluxes, even if limited by small sample size. Lansbury et al. (2017) find $f_{\mathrm{CT}} \sim 0.3$ at $z \lesssim 0.1$ down to $F_{10-40} \sim 10^{-13} \mathrm{erg} \mathrm{cm}^{-2} \mathrm{~s}^{-1}$. At intermediate redshift $(z \sim 0.5)$, Civano et al. (2015) found an observed $f_{\mathrm{CT}} \sim$ 0.2 , while Zappacosta et al. (2018) found an intrinsic $f_{\mathrm{CT}}$ between 0.1 and0.56. Finally, Masini et al. (2018) derived an observed $f_{\mathrm{CT}}=$ $0.11 \pm 0.02$ down to $F_{10-40} \sim 10^{-13}$ at $z \sim 1$.

Therefore, despite their expected intrinsic large fraction, CT AGN are very difficult to identify beyond the local Universe, resulting in a small/negligible number of CT AGN blindly identified in medium/deep X-ray surveys (e.g. Tozzi et al. 2006; Comastri et al. 2011; Georgantopoulos et al. 2013; Lanzuisi et al. 2013; Civano et al. 2015, Marchesi et al. 2016b, but see Brightman et al. 2014; Buchner et al. 2014, for a different approach).

Here we present the selection of 67 CT AGN candidates among the 1855 extragalactic sources with spectral analysis from the Chandra Cosmological Evolution Survey (COSMOS) Legacy catalogue (Marchesi et al. 2016b, hereafter M16). Section 2 describes the sample selection and spectral analysis. In Section 3, we explore the relation between X-ray luminosity (observed and intrinsic) and infrared (IR) luminosity. Section 4 presents the number counts of CT AGN in three redshift bins. In Section 5, we derive the intrinsic $f_{\mathrm{CT}}$ as a function of luminosity and redshift, and in Section 6, we exploit the Hubble Space Telescope (HST)-Advanced Camera for Surveys (ACS) coverage in the COSMOS field to derive merger fraction for CT AGN in three luminosity bins. Section 7 discusses these results.

\section{SAMPLE SELECTION}

\subsection{The COSMOS Survey}

The $2 \mathrm{deg}^{2}$ area of the HST COSMOS Treasury program is centred at 10:00:28.6, +02:12:21.0 (Scoville et al. 2007). The field has an unrivalled deep and wide multiwavelength coverage, from the optical band [Hubble, Subaru, Very Large Telescope (VLT), and other ground-based telescopes] to the infrared (Spitzer, Herschel), X-ray (XMM-Newton, Chandra, and NuSTAR) and radio bands [Very Large Array (VLA) at 1.4 and $3 \mathrm{GHz}$ and Very Long Baseline Array (VLBA)]. Large dedicated ground-based spectroscopic programs with all the major optical telescopes have been completed. Very accurate photometric redshifts are available for both the galaxy (Ilbert et al. 2009) and the AGN population (Salvato et al. 2011; Marchesi et al. 2016a).

The COSMOS field has been observed in X-rays with $X M M$ Newton for a total of $\sim 1.5 \mathrm{Ms}$ at a rather homogeneous depth of $\sim 60$ ks over $\sim 2 \operatorname{deg}^{2}$ (Hasinger et al. 2007; Cappelluti et al. 2009; Brusa et al. 2010), and by Chandra with deeper observations of $\sim 160$ ks: the central $\mathrm{deg}^{2}$ was observed in 2006-2007 (Elvis et al. 2009; Civano et al. 2012) for a total of $1.8 \mathrm{Ms}$, while additional $1.2 \mathrm{deg}^{2}$ were covered more recently (2013-2014) by the Chandra COSMOS Legacy Survey for a total of 2.8 Ms (Civano et al. 2016). 56 per cent of the Chandra detected sources have a spectroscopic redshift (Marchesi et al. 2016a).

We started from the results of the spectral analysis of the full Chandra COSMOS Legacy catalogue performed in M16. Their catalogue contains 1855 extragalactic sources with more than 30 net counts in the full $0.5-8 \mathrm{keV}$ band. This threshold allows the derivation of basic spectral properties $\left(N_{\mathrm{H}}, L_{\mathrm{X}}\right.$; see Lanzuisi et al. 2013). For each of them a simple spectral fit was performed in M16, including a power law modified by a local neutral absorber, fixed to the Galactic value in the direction of the field, plus a variable neutral absorber at the source redshift. The power-law photon index was left free to vary only for sources with more than 70 net counts due to the degeneracy between this parameter and $N_{\mathrm{H}}$.

In 67 cases a second power law was needed at 90 per cent confidence level (c.l., i.e. with an improvement of the fit of $\Delta \mathrm{C}$-stat $>$ 2.71; see also Tozzi et al. 2006; Brightman et al. 2014) in order to reproduce the emission emerging in the soft band above the obscured primary power law, while in 141 sources an emission line was needed at the same c.l. to reproduce the Fe $\mathrm{K} \alpha$ line at $6.4 \mathrm{keV}$. Fig. 1 shows the distribution of $N_{\mathrm{H}}$ versus photon index for the 1855 sources, divided on the basis of the optical/spectral energy distribution (SED) classification: red for type-2s, blue for type-1s, and green for galaxies. This classification is based on the presence or lack of broad emission lines in the optical spectra when available, or on the SED best-fitting template class (see M16 for details).

The procedure adopted in M16 is not optimized to look for CT AGN, and indeed only five sources were found to be in the CT regime. In general, simple models such as a single power law modified by photoelectric absorption are not able to correctly identify CT AGN, because:

(i) neutral, photoelectric absorption components, such as wABS or similar in XSPEC (Arnaud 1996), do not take into account Compton scattering, which becomes important above a few $\times 10^{23} \mathrm{~cm}^{-2}$, and do not allow the modelling of a realistic absorber geometry;

(ii) highly obscured spectra can be well reproduced also with a flat power law, $\Gamma \lesssim 1.4$ (George \& Fabian 1991; Georgantopoulos et al. 2011a), typically having a low $N_{\mathrm{H}}$ value. In this case the power 


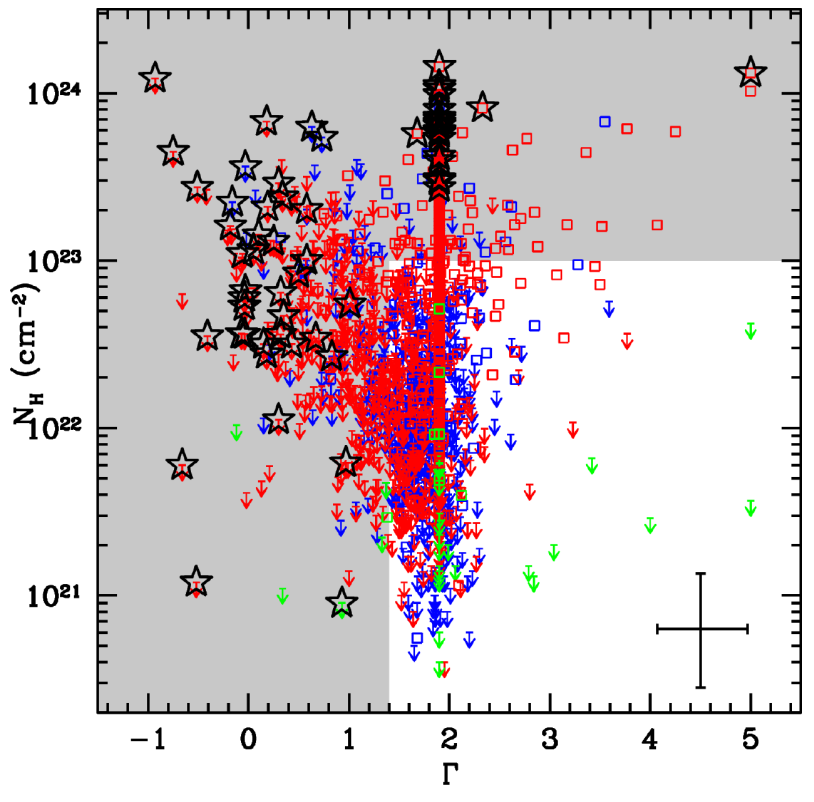

Figure 1. Distribution of photon index versus column density derived in M16 for the 1855 extragalactic sources with more than 30 net counts, colour coded for optical type (blue type-1, red type-2, and green galaxies). Squares represent $N_{\mathrm{H}}$ detections, arrows are 90 per cent upper limits. The grey area shows the search region for CT candidates: sources in this area were reanalysed with the physical model described in Section 2.2, and the $N_{\mathrm{H}}$ probability distribution function (PDF) was derived. The black stars highlight sources that have at least 5 percent of their $N_{\mathrm{H}} \mathrm{PDF}$ above the CT threshold, $10^{24} \mathrm{~cm}^{-2}$. The few sources with $\Gamma \gtrsim 4$ are star-forming galaxies with thermal X-ray spectra, or obscured sources with large errors in both $N_{\mathrm{H}}$ and $\Gamma$.

law reproduces the flat continuum typical of reflection-dominated sources, and the derived $N_{\mathrm{H}}$ can be heavily underestimated.

Therefore sources with $N_{\mathrm{H}}$ above $10^{23} \mathrm{~cm}^{-2}$ and/or photon index below 1.4 are candidate highly obscured sources and their X-ray spectra need to be properly modelled in order to retrieve a more accurate $N_{\mathrm{H}}$ estimate. In the next section we describe our novel approach (see also Akylas et al. 2016) that combines physically motivated models, such as MYTORUs (MY09), with Markov chain Monte Carlo (MCMC) parameter estimation techniques, and the use of the full PDF of the column density, to select CT AGN.

\subsection{X-ray modelling}

We reanalysed all the 662 sources in the grey-shaded area of Fig. 1, with the physical model MYTORUs that self-consistently takes into account photoelectric absorption, Compton scattering, cold reflection, and fluorescent emission in a fixed toroidal geometry. This model has several free parameters, and given the limited quality of the data available here, we decided to fix some of them.

The inclination angle between the line of sight and the axis of the torus, $\Theta_{\mathrm{obs}}$, is fixed to $75^{\circ}$ (where $\Theta_{\mathrm{obs}}=90^{\circ}$ corresponds to an edge-on observing angle) to ensure that the primary continuum is observed through the obscuring torus. Adopting inclination angles of $65^{\circ}$ or $90^{\circ}$, the two extremes for the torus intercepting the line of sight, translates into a typical $\Delta \log \left(N_{\mathrm{H}}\right)$ of +0.3 and -0.1 , respectively. The power-law photon index is fixed to the canonical value 1.8 (Piconcelli et al. 2005) to obtain tighter constraints on the parameter $N_{\mathrm{H}}$. A difference of \pm 0.3 in the assumed $\Gamma$ translates into a typical $\Delta \log \left(N_{\mathrm{H}}\right)$ of \pm 0.1 (see Appendix B for a more detailed analysis of the impact of changes in these two parameters on the derived $N_{\mathrm{H}}$ distribution).

The relative normalizations of the three MYTORUS components, i.e. the absorbed power law, the reflection, and the emission line complex, are fixed to 1 , i.e. the relative strength of the different components is fixed to the value derived for the geometry, covering factor, and element abundances adopted in MY09, and no continuum variability is allowed (the 'coupled version' of the model).

The model is therefore very simple, as it uses a single geometry, corresponding to a torus half-opening angle of $60^{\circ}$, for all sources, and does not allow for any variation between the primary continuum and the reflection/emission line component, or for different values of $N_{\mathrm{H}}$ between the absorber along the line of sight and the reflecting medium, as would be possible in the decoupled version of the model. This choice is forced by the very limited number of counts available for each source (65 per cent of the final CT candidate sample has less than 50 net counts).

In addition to the MYTORUs components, we added a secondary power law, with the photon index fixed to 1.8 , to model the emission emerging in the soft band in most of the obscured spectra (e.g. Lanzuisi et al. 2015). The normalization of this component is bound to be $<5$ per cent of the primary component, the typical limit for obscured local AGN (e.g. Noguchi et al. 2010). ${ }^{2}$

The definition of CT AGN implies a sharp threshold in hydrogenequivalent column density (in the literature typically assumed $N_{\mathrm{H}}>$ $10^{24} \mathrm{~cm}^{-2}$, or formally $N_{\mathrm{H}}>\sigma_{\mathrm{T}}^{-1} \sim 1.6 \times 10^{24} \mathrm{~cm}^{-2}$ ). The $N_{\mathrm{H}}$ parameter typically has large uncertainties in faint, high-redshift sources detected in deep surveys such as COSMOS (see Appendix A). Therefore, selecting CT sources based on $N_{\mathrm{H}}$ best-fitting value alone is subject to uncertainties and variation from one analysis to the other (see e.g. Castelló-Mor et al. 2013).

We adopt a different approach in our search for CT AGN. We performed the spectral analysis with XSPEC v. 12.9.1, using the Cash statistic (Cash 1979) with the direct background subtraction option (W-stat; Wachter, Leach \& Kellogg 1979). The spectra are binned to 3 counts bin $^{-1}$. Once the best fit with the standard W-stat likelihood is obtained, we run an MCMC within XSPEC, using the GoodmanWeare algorithm (Goodman \& Weare 2010) with $10^{4}$ steps to efficiently explore the parameter space. The full representation of the parameter space can be then marginalized to look separately at each parameter distribution and derive the full PDF.

With this method it is possible to properly account for cases in which the PDF has multiple peaks, as it is sometime the case for CT AGN candidates, where two solutions are similarly allowed by the data, one at lower $N_{\mathrm{H}}$ and lower intrinsic flux and one at higher $N_{\mathrm{H}}$ and flux (see e.g. Buchner et al. 2014). Fig. A1 shows an example of such double-peaked PDF in the parameter space $N_{\mathrm{H}}$ versus intrinsic flux for source lid_3516. The standard methods for error estimation would fail to correctly estimate the uncertainty in these cases, ignoring one of the two solutions.

Thanks to this analysis we were able to select a sample of 67 obscured sources, at $0.04<z<3.46$, that have at least 5 per cent of their $N_{\mathrm{H}}$ PDF above $10^{24} \mathrm{~cm}^{-2}$. Black stars in Fig. 1 show the $\mathrm{CT}$ candidates selected in this way. As hypothesized above, a large number of CT candidates have a flat power law $(\Gamma<1.4)$ with mild or negligible obscuration as best fit in the M16 catalogue. On the contrary, a number of sources with $N_{\mathrm{H}} \gtrsim 10^{23} \mathrm{~cm}^{-2}$ and steep

\footnotetext{
${ }^{2}$ This secondary power law must incorporate the redshift information (e.g. ZPOWERLW in XSPEC) so that the normalization, defined at $1 \mathrm{keV}$ rest frame, can be directly compared with the one of MYTORUS.
} 
power law do not have any significant fraction of the $N_{\mathrm{H}}$ PDF above $10^{24} \mathrm{~cm}^{-2}$ once the MYTORUs model with $\Gamma=1.8$ is adopted.

Fig. 2 shows the unfolded spectra plus residuals and the $N_{\mathrm{H}}$ PDF for three CT candidates selected in this way (Fig. D1, available in the online journal, shows spectra and $N_{\mathrm{H}} \mathrm{PDF}$ for the entire sample). The left-hand panel shows a C-thin source with a small PDF fraction above the CT threshold, the central panel shows a double-peaked $\mathrm{PDF}$ with one solution in the $\mathrm{C}$-thin regime and one in the heavily CT regime (see also Appendix A, Section A1), while the right-hand panel shows a heavily CT, reflection-dominated source. The upper boundary of $N_{\mathrm{H}}$ at $10^{25} \mathrm{~cm}^{-2}$ is set by the limit of the model.

If only the part of the PDF above the CT threshold (red area) is taken into account when computing the number of $\mathrm{CT}$, it is possible to construct an effective sample of CT AGN candidates, by counting each source only for the fraction of the PDF that exceeds the CT boundary. This means that virtually none of these sources have 100 per cent probability of being CT or C-thin, but we can consider the sum of the probabilities above the CT threshold for the whole sample as a good approximation of the total number of CT in that sample. Summing up only the fraction of the PDF of each source that is above $10^{24} \mathrm{~cm}^{-2}$, we obtained a number of CT sources of $N_{\mathrm{CT}}=$ 38.5. This number is stable with respect to the threshold adopted to select CT AGN, as it would be 38.1 or 38.8 if this threshold is taken at 10 or 1 per cent of the PDF above $10^{24} \mathrm{~cm}^{-2}$, respectively.

Table 1 summarizes the results for all the 67 CT AGN candidates. All our sources have a $0.5-7 \mathrm{keV}$ signal-to-noise ratio $(\mathrm{SNR})>$ 4, despite having typically a low number of counts (column 3), thanks to the very low background levels of Chandra. The sources

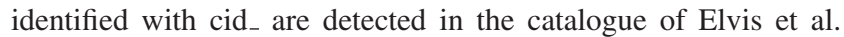
(2009) and Civano et al. (2012), while the ones identified with lid come from the catalogue of Civano et al. (2016) and Marchesi et al. (2016a). For 60 percent of the sample a spectroscopic redshift is available. The remaining sources have photometric redshift, with good accuracy. The errors reported in column (2) are the 90 per cent c.l. errors computed from the photometric redshift PDF. The vast majority of them are $<0.10$, a few up to $\sim 0.5$ and only three sources (lid_4053, cid_1054, and cid_2177) have errors larger than 0.9 due to the presence of secondary peaks of the PDF distribution.

We note that four sources at $z<1$ (labelled with ${ }^{a}$ in Table 1) are detected also by the NUSTAR survey performed in COSMOS (Civano et al. 2015). The best-fitting $N_{\mathrm{H}}$ obtained by fitting XMMNewton and/or Chandra data together with NUSTAR (Zappacosta et al. 2018) is consistent, within the errors, with the one derived here, even if there is a tendency for slightly lower $N_{\mathrm{H}}$ obtained when NUSTAR is included in the fit (see Marchesi et al. 2018 for a systematic study of this effect on a sample of local CT AGN).

\section{$3 \quad L_{X}$ VERSUS $L_{I R}$}

One of the key parameters that can be derived from the X-ray spectral fitting of CT AGN is the intrinsic, absorption-corrected 2$10 \mathrm{keV}$ luminosity $\left(L_{\mathrm{X}}\right)$. To compute its value and realistic errors, we used the CFLUX component in XSPEC, applied to the unobscured, primary power law. ${ }^{3}$ In this way the intrinsic flux due to the primary power law becomes a free parameter of the fit, and its errors can

\footnotetext{
${ }^{3}$ The reflection and emission line components have their own cFLux applied, and their flux ratios with respect to the unabsorbed primary power law are fixed to the value derived from the MYTORUs model itself, i.e. 2.7 and 0.6 per cent, respectively, in the rest-frame $2-10 \mathrm{keV}$ band, in order to keep the proper relative normalization between the different components.
}

be evaluated self-consistently with the errors on $N_{\mathrm{H}}$, with the same MCMC approach. These values are then converted into $L_{\mathrm{X}}$ based on the luminosity distance of each source.

The sample of selected CT candidates spans a wide range in redshift, $0.04<z<3.46$, and absorption-corrected X-ray luminosity, $42<\log \left(L_{\mathrm{X}}\right)<45.7 \mathrm{erg} \mathrm{s}^{-1}$. Fig. 3 (left) shows the normalized redshift distribution for the CT AGN sample (orange) and the M16 sample (cyan). The thick lines show the distribution derived with a kernel density estimation (KDE) using a Gaussian kernel with a bandwidth of 0.3 applied to the underlying distributions for better visualization. The CT AGN tend to have a higher redshift (mean redshift 1.71, median 1.75) with respect to the M16 sample (mean 1.42 , median 1.29). This is a result of the positive $k$-correction for CT AGN in X-ray mentioned in Section 1. Fig. 3 (right) shows the same for the absorption-corrected $2-10 \mathrm{keV}$ luminosity. In this case the CT sample has one order of magnitude higher $L_{\mathrm{X}}$ (mean $\log \left(L_{\mathrm{X}}\right) 44.7$ versus $43.8 \mathrm{erg} \mathrm{s}^{-1}$, median 44.8 versus 43.9 ) with respect to the M16 sample. In this case the difference is larger thanks to the larger correction for absorption applied in the CT sample with respect to the full catalogue.

Indeed, given the range of corrections applied for the obscuration, the majority of the sources in the CT sample are in the quasar regime $\left(L_{X}>10^{44} \mathrm{erg} \mathrm{s}^{-1}\right)$. In order to verify if these luminosities are consistent with other information available for this sample, i.e. if our estimates of the obscuration are correct, we compared the intrinsic X-ray luminosity with the mid-IR $(6 \mu \mathrm{m})$ AGN luminosity, as computed from the SED fitting from Delvecchio et al. (2015), or Suh et al. (2017) if the source is not far-IR (FIR) detected, after removing the host star formation emission in the same band. In total 54 out of 67 sources have this information available, while for the remaining 13, all not FIR detected, six have an SED fit in the analysis of Suh et al. (2017), but no significant torus component, and seven do not have the minimum of five photometric band detections required in Suh et al. (2017) to perform the SED fitting.

Fig. 4 shows the distribution of the AGN mid-IR luminosity versus the observed (left) and absorption-corrected (right) $L_{\mathrm{X}}$ for the sample of CT AGN (filled circles), compared with the 1048 sources in the COSMOS Legacy catalogue with $L_{\mathrm{IR}}^{\mathrm{AGN}}$ available (grey dots). The magenta curve shows the relation between intrinsic $L_{\mathrm{X}}$ and $L_{\mathrm{IR}}^{\mathrm{AGN}}$ published in Stern (2015). The dispersion around this relation for the M16 sample is $\sigma=0.38$.

It is generally assumed that CT AGN have the same distribution as the M16 sample. However, as can be seen from the right-hand panel, 90 per cent of the CT AGN selected here lie above the average $L_{\mathrm{X}}-L_{\mathrm{IR}}^{\mathrm{AGN}}$ relation, with an average offset of $0.7 \mathrm{dex}$, and there are seven sources that lie at about $3 \sigma$ from the relation. While for some of these sources it is possible that the correction for absorption is overestimated (notice also the large uncertainties on the intrinsic luminosities), Fig. 4 suggests that CT sources with intrinsic $L_{X}$ below the Stern (2015) relation are missed because their observed $L_{X}$ would cause them to be below the selection threshold applied here based on number of counts, or even below the detection threshold for the survey. Indeed there are 40 sources with 5-30 net counts and $L_{\mathrm{IR}}^{\mathrm{AGN}}$ available (not shown here) that lie below the curve for $\log N_{\mathrm{H}}=24$ in the left-hand panel, and that would be missing in the right-hand panel, since we do not have a reliable estimate of the intrinsic $L_{\mathrm{X}}$.

As a further proof of this, we used the properties of seven sources lying at $\sim 1 \sigma$ above the relation for the intrinsic $L_{\mathrm{X}}$ (from Fig. 4 , right-hand panel) to simulate what would be the observed count rate if such sources were at $1 \sigma$ below this relation (i.e. 0.8 dex lower intrinsic $L_{\mathrm{X}}$ ). These sources cover a range of $z$ and $N_{\mathrm{H}}$ representative 

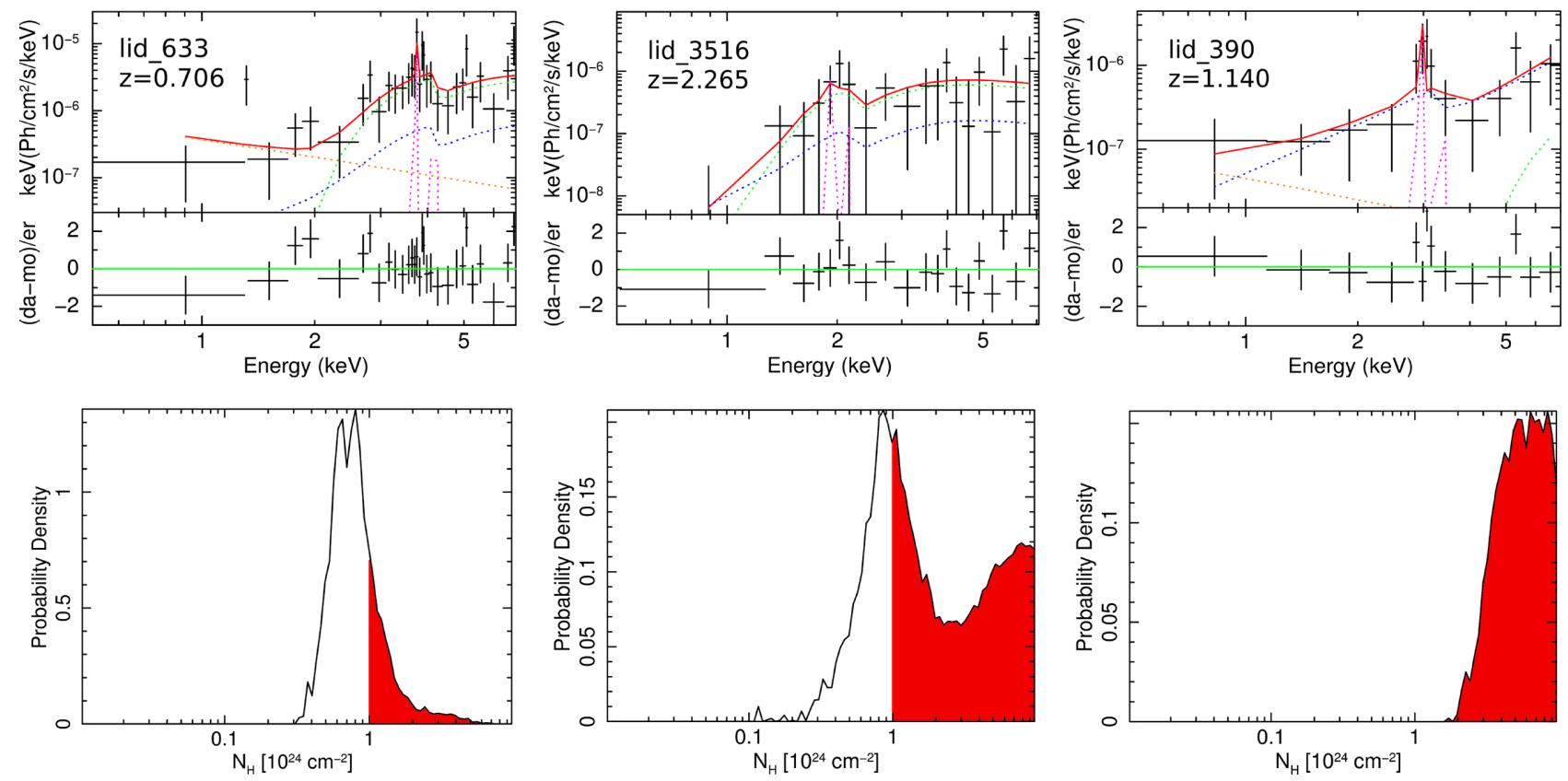

Figure 2. Top: unfolded spectrum and data-to-model ratio of the CT candidates lid_633, lid_3516, and lid_390 at $z=0.706,2.265$, and 1.140 , respectively. In green we show the obscured power law, in blue the reflection component, in magenta the emission lines component, and in orange the scattered power law emerging in the soft. The red curve shows the total best-fitting model. Bottom: PDF of $N_{\mathrm{H}}$ for the three sources shown above. The red area shows the fraction of $N_{\mathrm{H}}$ PDF above the CT threshold (column 5 in Table 1).

of the entire sample. The typical number of counts from the simulated spectra (all the parameters being the same with the exception of the intrinsic $L_{X}$ ) is $\sim 10$ net counts, with SNR $\sim 1.5$ for six out of seven sources, and in one case the simulated spectrum is below the background level. Therefore CT source $1 \sigma$ below the Stern (2015) relation would be excluded from our analysis due to a low number of counts, or even undetected in the COSMOS Legacy catalogue itself.

This suggests that a sizable fraction of CT AGN with similar properties but lower intrinsic luminosity, with respect to the detected ones, is still missing due to their low X-ray fluxes. This selection effect, due to the flux limit of the survey, will be taken into account (see Appendix C) when computing intrinsic CT fractions.

We can also derive a selection efficiency in the region below the cyan dotted line in the left-hand panel of Fig. 4 (i.e. observed $L_{X}$ below what expected for $\log N_{\mathrm{H}}=24 \mathrm{~cm}^{-2}$ ), which is often adopted in the literature for selection of CT candidates.

While in the local Universe it is possible to sample $L_{\mathrm{X}}-L_{\mathrm{IR}}^{\mathrm{AGN}}$ offsets of up to 2-3 dex ( Gandhi et al. 2009; Asmus et al. 2015) making this selection effective (see also La Caria et al. 2018), in high- $z$ surveys the depth of the X-ray observation does not allow to sample such high $L_{\mathrm{X}}-L_{\mathrm{IR}}^{\mathrm{AGN}}$ offsets, translating into a limited efficiency (see e.g. Lanzuisi et al. 2009; Georgantopoulos et al. 2011) or large fractions of non-detection ( Alexander et al. 2008; Del Moro et al. 2016; see Goulding et al. 2011 for a low- $z$ example on shallow X-ray data).

Indeed we can show that the efficiency of CT selection for our sample is only $\sim 33$ percent (5.3 effective CT candidates among 16 total sources below the selection threshold), while it misses 90 per cent of the CT candidates selected on the basis of the spectral analysis. Therefore, the selection of CT AGN based only on the distance from the intrinsic relation, in high- $z$ samples in mediumdeep X-ray surveys, is not efficient because of the large dispersion in the intrinsic relation and because CT sources lying below the intrinsic relation tend to be not detected in the X-ray catalogue.

\section{NUMBER DENSITY OF CT AGN}

Taking advantage of the large sample of CT AGN selected through the analysis described in Section 3, we derived number counts for our CT AGN sample in three redshift bins: $z=0.04-1, z=1-$ 2 , and $z=2-3.5$ (hereafter $z 1, z 2$, and $z 3$, respectively). Each source is counted only for the fraction of PDF above $10^{24} \mathrm{~cm}^{-2}$. We apply the corrections described in Appendix C (Sections C1 and $\mathrm{C} 2$, differential sky coverage and classification bias, respectively), computed as a function of source redshift and flux.

The $\log N-\log S$ in the three bins is shown in Fig. 5. The error in each data point is computed taking into account only the Poissonian error related to the number of sources observed. We compare our data points with the model predictions from Akylas et al. (2012). In this model it is possible to modify the intrinsic fraction of CT, $f_{\mathrm{CT}}$, together with other parameters such as power-law photon index, high energy cut-off $\left(E_{\text {cut }}\right)$, and reflection fraction $\left(f_{\mathrm{R}}\right)$, expressed as the ratio between reflection and intrinsic continuum fluxes in the 2-10 keV rest-frame band. ${ }^{4}$

From this model, we derived the $\log N-\log S$ for sources in the $N_{\mathrm{H}}$ bin 24-26 assuming $\Gamma=1.8, E_{\text {cut }}=195 \mathrm{keV}, f_{\mathrm{R}}=0.03$ (consistent with the reflection fraction of $\sim 3$ percent derived from the model described in Section 2.2) in the three redshift bins. Keeping all the other parameters constant, $f_{\mathrm{CT}}$ must increase from 0.3 in $\mathrm{z} 1$, to 0.45 and 0.55 in z 2 and z3, respectively, to match the observed distribution at those redshifts. All these values of $f_{\mathrm{CT}}$ are still within the $1 \sigma$ c.l. contours for the poorly constrained fraction of CT AGN derived

\footnotetext{
${ }^{4}$ See the online tool at http://indra.astro.noa.gr/xrb.html
} 
Table 1. Spectral properties of the CT AGN candidates. Column (1): source ID from Civano et al. (2016); column (2): redshift (photometric ones are reported with 90 per cent c.l. errors); column (3): net $0.5-7 \mathrm{keV}$ counts; column (4): signal-to-noise ratio (SNR); column (5): fraction of PDF above $N_{\mathrm{H}}=10^{24} \mathrm{~cm}^{-2}$ (corrected for classification bias); column (6): best-fitting $\log \left(N_{\mathrm{H}}\right)$ in $\mathrm{cm}^{-2}$; column (7): scatter fraction in per cent; column (8): observed 2-10 keV flux; column (9): observed 2-10 keV luminosity; column (10): absorption-corrected 2-10 keV luminosity; column (11): bolometric luminosity; and column (12): C-stat/d.o.f. of the best fit.

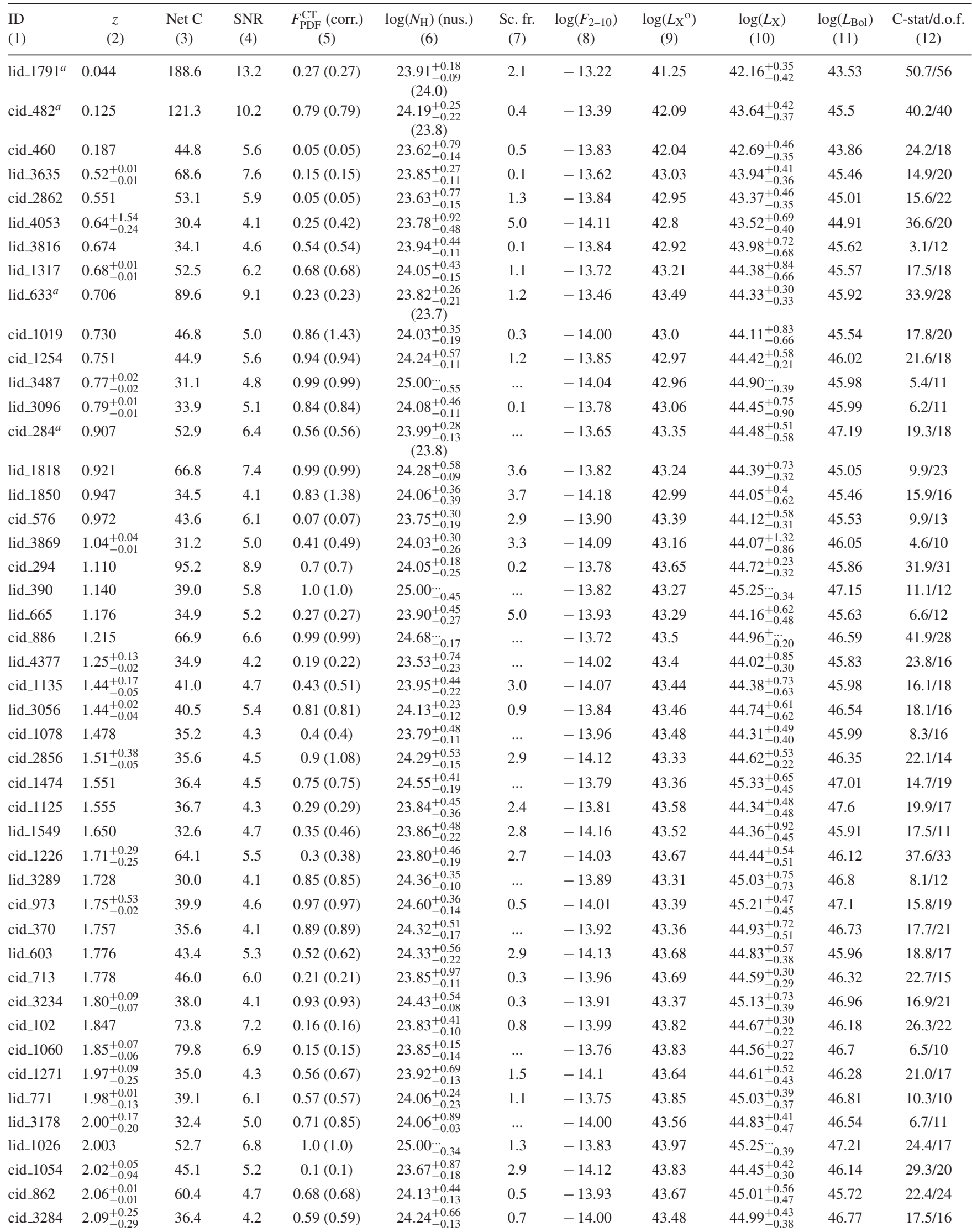


Table 1 - continued

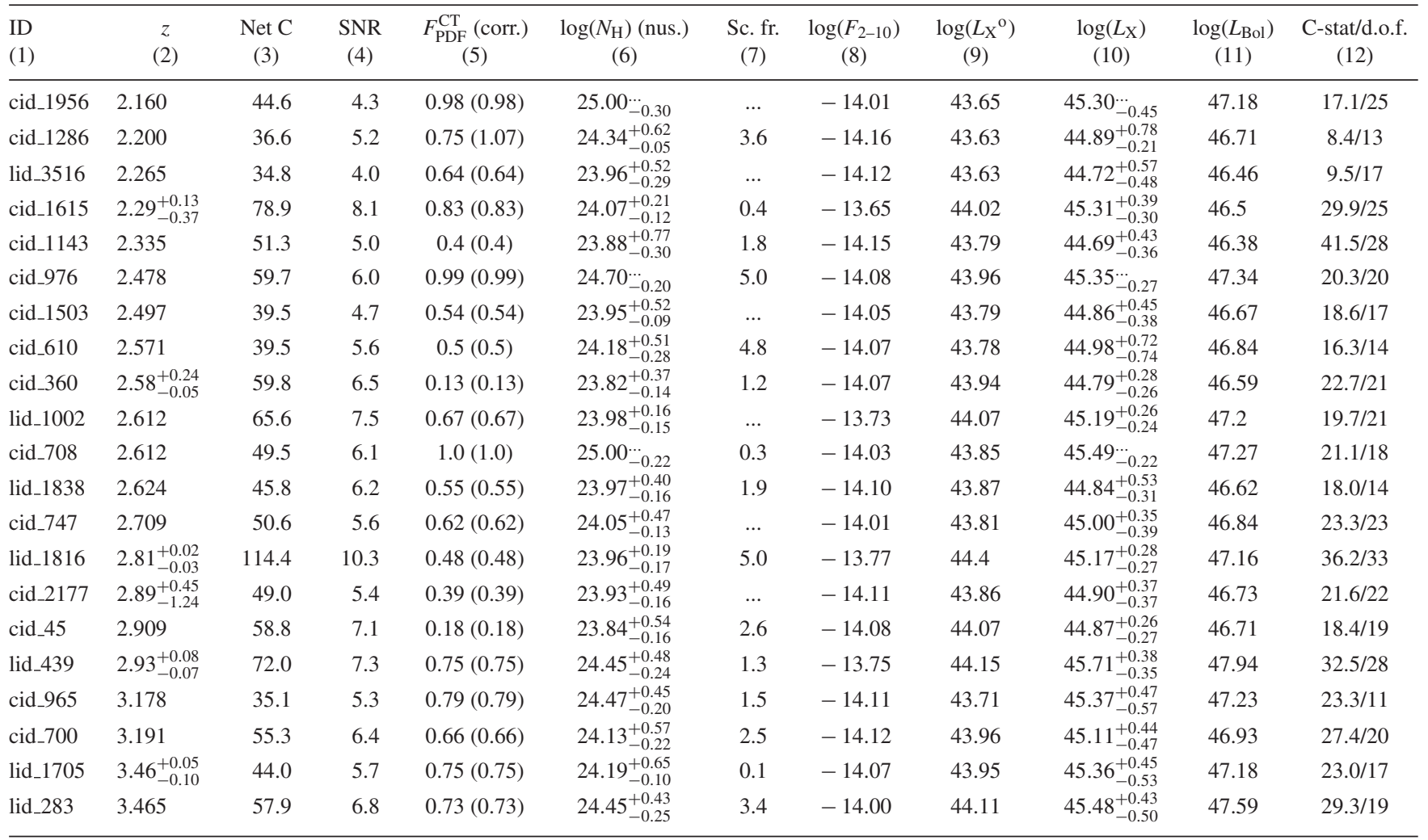

Note. Errors in columns (6) and (9) are at 90 per cent c.l. The $N_{\mathrm{H}}$ value (from Zappacosta et al. 2018) is in parenthesis in column (6).

${ }^{a}$ Sources detected with NUSTAR.
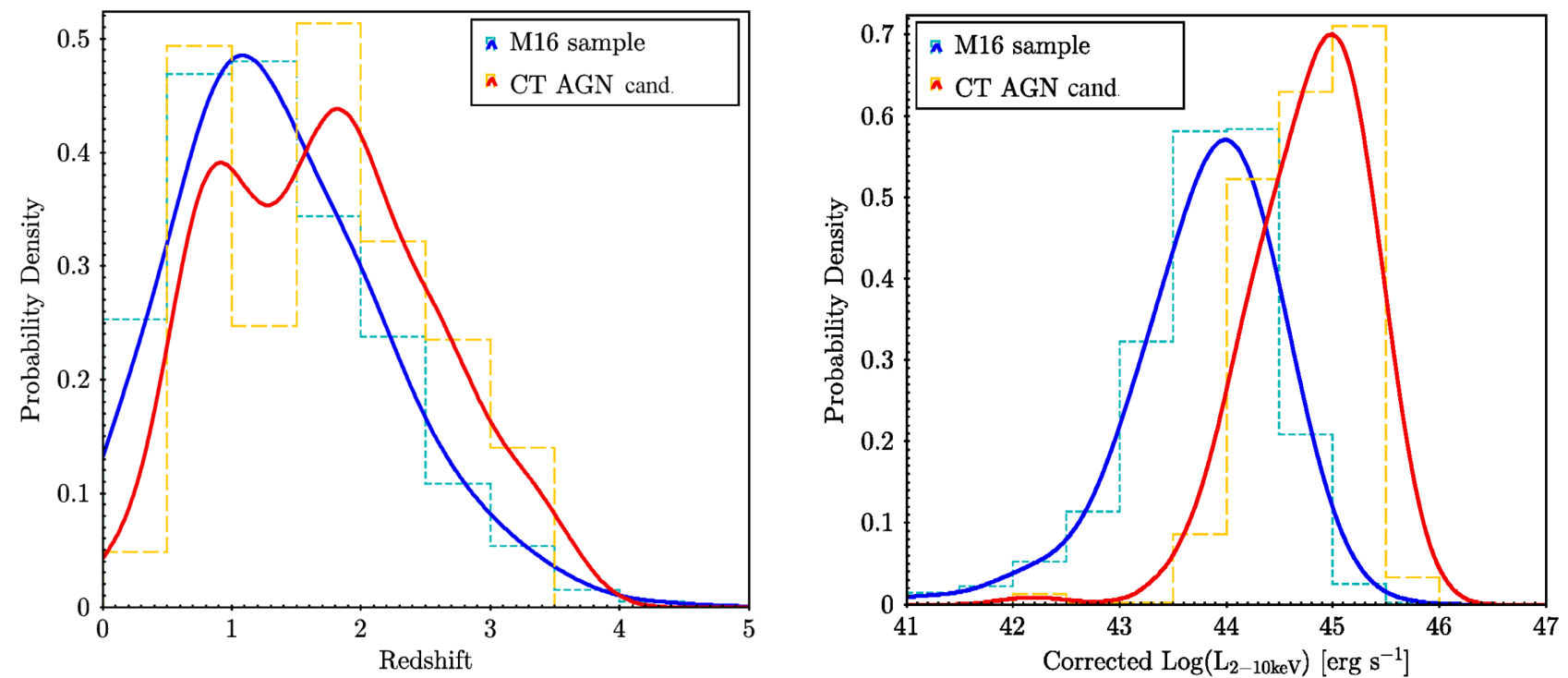

Figure 3. Redshift (left) and absorption-corrected 2-10 keV band luminosity (right) normalized distributions for the CT AGN candidates (red) and the M16 sample (blue). The CT sample is corrected for classification bias and survey sensitivity, and each source is counted only for the fraction of the PDF above the CT threshold. The thick lines show the distribution derived with a kernel density estimation (KDE) using a Gaussian kernel with a bandwidth of 0.3 applied to the underlying distributions (dashed histograms). The CT AGN tend to have higher redshift with respect to the M16 sample, while the typical absorption-corrected 2-10 keV luminosity is one order of magnitude higher (see text).

from the Akylas et al. (2012) fit for the X-ray background (XRB) intensity as a function of energy. A more quantitative estimate of the evolution of $f_{\mathrm{CT}}$ with redshift will be derived in Section 5 using a different approach.
Using the $\log N-\log S$ from the full COSMOS Legacy catalogue (Civano et al. 2016), we can derive the observed fraction of CT AGN as a function of observed $2-10 \mathrm{keV}$ flux. This quantity is useful since it allows for comparisons with both data points from 

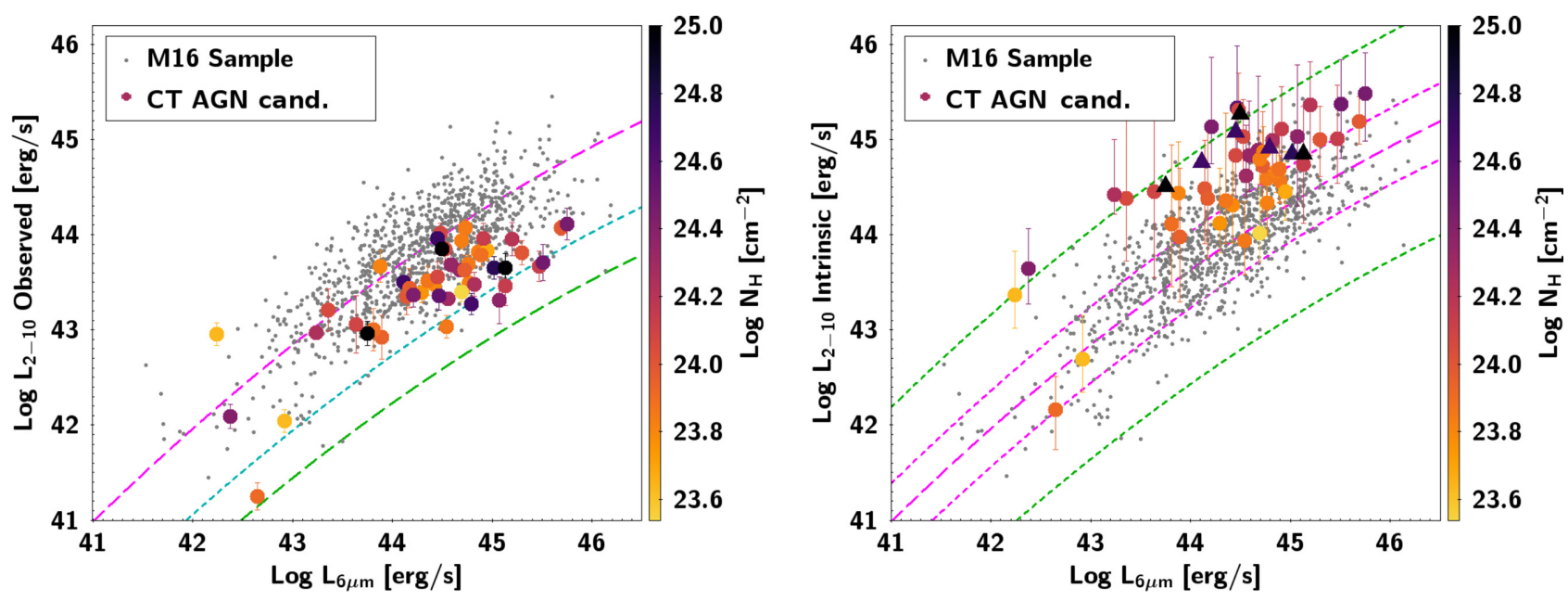

Figure 4. Distribution of the AGN mid-IR (6 $\mu \mathrm{m})$ luminosity, as derived from SED fitting, versus $2-10 \mathrm{keV}$ luminosity for the 1048 sources in the COSMOS Legacy catalogue with $L_{\mathrm{IR}}^{\mathrm{AGN}}$ available (grey dots). Left-hand panel shows the observed $L_{\mathrm{X}}$, while right-hand panel shows the absorption-corrected $L_{\mathrm{X}}$. Data points for the CT candidates are colour coded on the basis of $\log N_{\mathrm{H}}$. Triangles show lower limits of the intrinsic $L_{\mathrm{X}}$ for sources with a lower limit in the $N_{\mathrm{H}}$. The magenta dashed curve in both panels shows the relation from Stern (2015). In the left-hand panel, the cyan dotted (green dashed) line shows the $L_{\mathrm{X}}$ decrement expected for $\log N_{\mathrm{H}}=24\left(\log N_{\mathrm{H}}=25\right) \mathrm{cm}^{-2}$, assuming the model described in Section 2.2. In the right-hand panel, the dotted magenta (green) curves show the $1 \sigma(3 \sigma)$ dispersion of the COSMOS Legacy sample.

the literature and CXB models. Fig. 6 shows the observed $f_{\mathrm{CT}}$ in three flux bins for the COSMOS Legacy sample (red circles). The orange curve shows the prediction from the baseline CXB model of Ueda et al. (2014), which derives an intrinsic fraction $N(24-26) / N(20-26)$ of $\sim 0.33$. The blue curve shows the G07 model, with an intrinsic $N(24-26) / N(20-26)$ of $\sim 0.37$ averaged over all luminosities. The cyan and green curves show two different realizations of the Akylas et al. (2012) model. Both have $\Gamma=1.8$ and $E_{\text {cut }}=195 \mathrm{keV}$, while $f_{\mathrm{R}}$ goes from 0.03 (cyan) to 0.05 (green) and $f_{\mathrm{CT}}$ from 0.5 (cyan) to 0.3 (green). None of these models, however, allow for any evolution of the $f_{\mathrm{CT}}$ with redshift.

Note that the increase from $f_{\mathrm{R}}=0.03$ (cyan) to 0.05 (green) compensates for the decrease in $f_{\mathrm{CT}}$, especially at the bright fluxes, and therefore both realizations of the Akylas et al. (2012) model are in agreement with our data points. The cyan curve (lower $f_{\mathrm{R}}$ and higher $f_{\mathrm{CT}}$ ) is in better agreement with the lowest flux data point coming from Chandra Deep Field-South (CDF-S) 4 Ms (BU12), while the green curve (higher $f_{\mathrm{R}}$ and lower $f_{\mathrm{CT}}$ ) is in better agreement with other data points from the literature at higher fluxes. We note that the flux ratio between direct and reflected components in the 2$10 \mathrm{keV}$ band, derived from the MYTORUS model, with the parameters described in Section 2, and $\log \left(N_{\mathrm{H}}\right)=24\left(\mathrm{~cm}^{-2}\right)$ is 0.033 , close to the values for the cyan curve.

The discrepancy of the observed $f_{\text {СT }}$ predicted by different models at high fluxes is mainly due to the different assumptions on the reflection component: in G07 the reflection contribution is different for obscured and unobscured sources, and is neglected for luminous sources. In Akylas et al. (2012), $f_{\mathrm{R}}$ is the same for all sources. We also add that in both cases, the reflection component is modelled with a disc-reflection model (i.e. PEXRAV in XSPEC), while for CT sources the reflection produced by a toroidal structure would be more appropriate and produce a different spectral shape (see MY09).

\section{INTRINSIC FRACTION OF CT AGN}

We derive the intrinsic fraction of CT AGN in three redshift bins, in a common $L_{X}$ range, following the procedure described in BU12. In order to compute intrinsic fractions of CT sources in $z$ and $L_{\mathrm{X}}$ bins, we have to build samples that are complete with respect to a given value of $N_{\mathrm{H}}$. Therefore we compute the sensitivity curves shown in Fig. 7, by converting the flux limit of our CT sample, $\log F_{2-10 \mathrm{keV}}>-14.2\left(\mathrm{erg} \mathrm{cm}^{-2} \mathrm{~s}^{-1}\right)$, into an intrinsic luminosity limit, for a given redshift, computed adopting a spectral model as described in Section 2.2, and with $\log \left(N_{\mathrm{H}}\right)=24,24.5$, and $25\left(\mathrm{~cm}^{-2}\right)$, respectively.

All the sources from the full COSMOS Legacy catalogue above each of the three curves shown in Fig. 7 constitute a complete sample up to that $N_{\mathrm{H}}$. Unfortunately, the curve for $\log \left(N_{\mathrm{H}}\right)=25\left(\mathrm{~cm}^{-2}\right)$ includes very few sources, and therefore the determination of the intrinsic $f_{\mathrm{CT}}$ would have very large uncertainties. We therefore decided to use the complete sample above the curve for $\log \left(N_{\mathrm{H}}\right)=$ $24.5\left(\mathrm{~cm}^{-2}\right)$, and computed the fraction of CT AGN using the samples (in each redshift bin) above the curve for $\log \left(N_{\mathrm{H}}\right)=24.5 \mathrm{~cm}^{-2}$. This represents a lower limit of the full intrinsic $f_{\mathrm{CT}}$ defined as $N\left(\log N_{\mathrm{H}}=24-25\right) / N($ tot $)$, since we can still miss some sources with $\log \left(N_{\mathrm{H}}\right)>24.5 \mathrm{~cm}^{-2}$ close to the completeness curve. The fractions that are obtained for the sample above $\log \left(N_{\mathrm{H}}\right)=25\left(\mathrm{~cm}^{-2}\right)$ are however very similar, although with large error bars, in all the three $z$ bins. Therefore we argue that the $f_{\mathrm{CT}}$ derived for the sample complete up to $\log \left(N_{\mathrm{H}}\right)=24.5\left(\mathrm{~cm}^{-2}\right)$ is a good approximation for the full CT sample.

Table 2 summarizes the results obtained following this approach. The number of CT AGN is corrected accounting for the classification bias and survey sensitivity. The average $\log L_{\mathrm{X}}$ and its dispersion (at $1 \sigma$ c.l.) are derived from the full sample (CT+C-thin) in each redshift bin. Because of the small sample sizes we adopted 

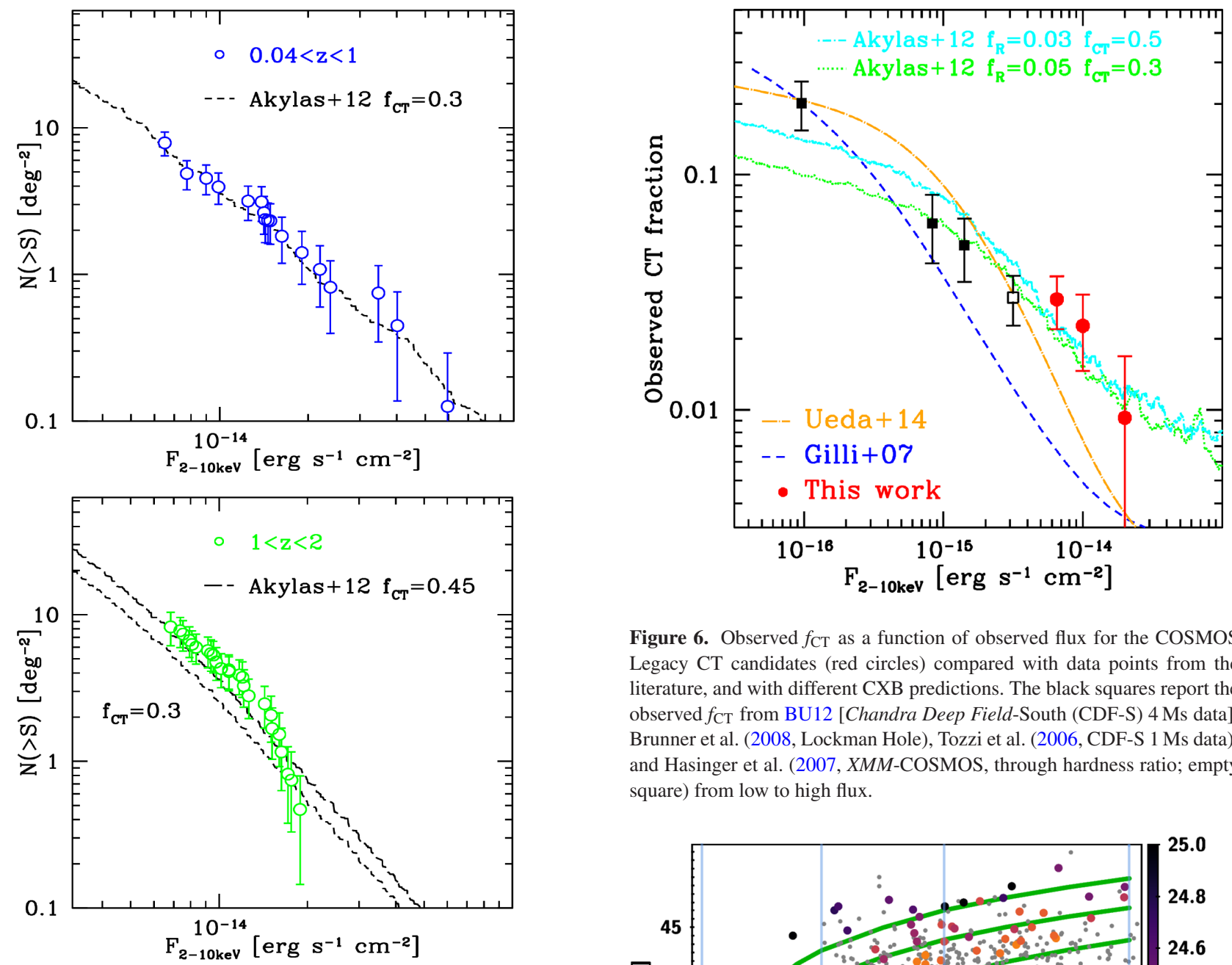

Figure 6. Observed $f_{\mathrm{CT}}$ as a function of observed flux for the COSMOS Legacy CT candidates (red circles) compared with data points from the literature, and with different $\mathrm{CXB}$ predictions. The black squares report the observed $f_{\mathrm{CT}}$ from BU12 [Chandra Deep Field-South (CDF-S) 4 Ms data], Brunner et al. (2008, Lockman Hole), Tozzi et al. (2006, CDF-S 1 Ms data), and Hasinger et al. (2007, XMM-COSMOS, through hardness ratio; empty square) from low to high flux.

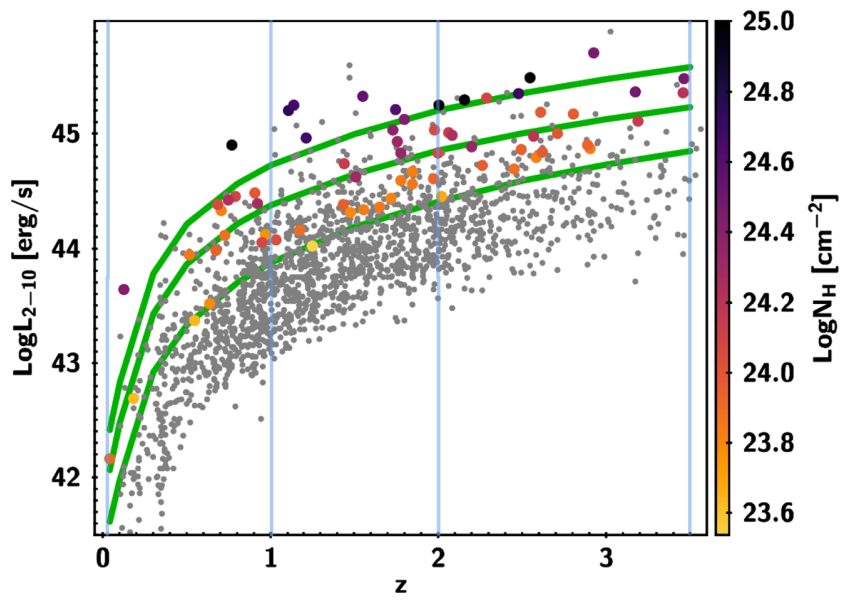

Figure 7. Distribution of intrinsic 2-10 keV luminosity versus redshift for the CT AGN candidates (colour coded for $\log \left(N_{\mathrm{H}}\right)$ ), and for the sample in M16 (grey dots). The vertical blue lines mark the limits of the three redshift bins. The green curves show the limit of intrinsic luminosity we are sensitive to, for $\log \left(N_{\mathrm{H}}\right)=24,24.5$, and $25\left(\mathrm{~cm}^{-2}\right)$ given the survey flux limit.

the Bayesian approach presented in Cameron (2011) to derive confidence intervals (at $1 \sigma$ c.l.) on the observed ratio $f_{\mathrm{CT}}$.

To rescale $f_{\mathrm{CT}}$ to a common $L_{\mathrm{X}}$ range, we exploit the well-known 2 (centre), and $2<z<3.5$ (bottom), corrected for survey sensitivity and classification bias, compared with the model of Akylas et al. (2012). The short dashed curves show the expected $\log N-\log S$ for a constant $f_{\mathrm{CT}}=$ 0.3 , while the long dashed curves in the centre and bottom panel show the increased $f_{\mathrm{CT}}$ needed to qualitatively match the observed data points. linear relation between the fraction of obscured $\mathrm{AGN} f_{2}$, defined as $f_{2}=N(22-24) / N(20-24)$, and $\log \left(L_{\mathrm{X}}\right)$ (see e.g. Hasinger 2008; BN12; Ueda et al. 2014). As done in BN12 we adopted the slope $0.281 \pm 0.016$ found in Hasinger (2008) on a sample of $>1000$ AGN up to $z=5$. In the following we take into account the slope uncertainties in the extrapolation to a common $L_{\mathrm{X}}$ range. 
Table 2. Intrinsic $f_{\mathrm{CT}}$ at different luminosities for the three redshift bins. Column (1): redshift range; column (2): number of CT AGN above the log $\left(N_{\mathrm{H}}\right)=$ $24.5\left(\mathrm{~cm}^{-2}\right)$ completeness curve, after correcting for classification bias; column (3): surface density of CT AGN, given the survey flux-area curve; column (4): number of C-thin AGN above the $\log \left(N_{\mathrm{H}}\right)=24.5\left(\mathrm{~cm}^{-2}\right)$ completeness curve; column (5): surface density of C-thin AGN; column (6): average $L_{\mathrm{X}}$ and dispersion for the total sample; column (7): $f_{\mathrm{CT}}$ at the observed average $\log L_{\mathrm{X}}$; column (8): $f_{\mathrm{CT}}$ rescaled at $\log \left(L_{\mathrm{X}}\right)=44.5$ ( $\left.\mathrm{erg} \mathrm{s}^{-1}\right)$. The errors in this column also take into account the uncertainty on the slope F2 versus $L_{X}$.

\begin{tabular}{|c|c|c|c|c|c|c|c|}
\hline (1) & $\begin{array}{l}N_{\mathrm{CT}} \\
(2)\end{array}$ & $\begin{array}{c}N_{\mathrm{CT}} \\
\left(\mathrm{deg}^{-2}\right) \\
(3)\end{array}$ & $\begin{array}{r}N_{\text {thin }} \\
\text { (4) }\end{array}$ & $\begin{array}{c}N_{\text {thin }} \\
\left(\mathrm{deg}^{-2}\right) \\
(5)\end{array}$ & $\begin{array}{c}\left\langle\log \left(L_{X}\right)\right\rangle \\
(6)\end{array}$ & $\begin{array}{l}f_{\mathrm{CT}} \\
(7)\end{array}$ & $\begin{array}{c}f_{\mathrm{CT}}(44.5) \\
(8)\end{array}$ \\
\hline $0.04<z<1$ & 7.14 & 3.36 & 23 & 11.23 & $44.1 \pm 0.3$ & $0.24_{-0.07}^{+0.08}$ & $0.19_{-0.06}^{+0.07}$ \\
\hline $1<z<2$ & 8.67 & 4.29 & 25 & 11.80 & $44.9 \pm 0.2$ & $0.26_{-0.07}^{+0.08}$ & $0.30_{-0.08}^{+0.10}$ \\
\hline $2<z<3.5$ & 9.51 & 4.54 & 13 & 6.80 & $45.2 \pm 0.2$ & $0.42_{-0.09}^{+0.10}$ & $0.48_{-0.11}^{+0.12}$ \\
\hline
\end{tabular}

We derive $f_{\mathrm{CT}}$ (i.e. $\left.N(24-26) / N(20-26)\right)$ at a given $L_{\mathrm{X}}$ using the relation observed between $f_{2}$ and $L_{\mathrm{X}}$, assuming a flat $N_{\mathrm{H}}$ distribution above $\log \left(N_{\mathrm{H}}\right)=22 \mathrm{~cm}^{-2}$ (i.e. $N(24-26)=N(22-24)$, and therefore $f_{\mathrm{CT}}=f_{2} /\left(1+f_{2}\right)$. This assumption is supported by the fact that the $N_{\mathrm{H}}$ distribution, observed in the CT sample and control sample together, is indeed nearly flat in the bins $\log N_{\mathrm{H}}(22-24)-\log N_{\mathrm{H}}(24-$ 26) (we observe a ratio of 1.2:1.0:0.9 in the three redshift bins). For each redshift bin, we therefore use the average $L_{X}$ of the CT+C-thin sample, and extrapolate $f_{\mathrm{CT}}$ to $\log \left(L_{\mathrm{X}}\right)=44.5 \mathrm{erg} \mathrm{s}^{-1}$ in each bin using these relations.

The results of this approach are shown in Fig. 8 (left) for the three redshift bins. The filled squares represent the measured $f_{\mathrm{CT}}$ for each redshift bin, sampling increasing luminosities at increasing redshift. The empty squares are the values of $f_{\mathrm{CT}}$ at $\log \left(L_{\mathrm{X}}\right)=44.5 \mathrm{erg} \mathrm{s}^{-1}$ estimated with the method explained above.

We note that, given the definitions above, $f_{2}$ cannot be $>1$ (the extreme case in which all the $\mathrm{C}$-thin sources are obscured above $10^{22} \mathrm{~cm}^{-2}$ ) and therefore $f_{\mathrm{CT}}$ cannot be $>0.5$, by construction. In this regime the model is clearly an oversimplification; for example, it is possible that the relation $f_{2}-\log \left(L_{\mathrm{X}}\right)$ evolves with redshift, or that the assumption $N(24-26)=N(22-24)$ is no longer valid.

Fig. 8 (right) shows the evolution of $f_{\mathrm{CT}}$ with redshift, as derived in the left-hand panel, for $\log \left(L_{X}\right)=44.5 \mathrm{erg} \mathrm{s}^{-1}$ for the COSMOS Legacy sample (red empty squares). We compare our results with others from the literature: in particular, the blue error bars show the results from BU12 from the 4 Ms CDF-S data set at $\log \left(L_{X}\right)=$ $43.5\left(\mathrm{erg} \mathrm{s}^{-1}\right)$; magenta and cyan circles show the results from Ricci et al. (2015) from the hard-band-selected Swift-Burst Alert Telescope (BAT) sample in the local Universe ${ }^{5}$ at $\operatorname{low}\left(\log L_{14-195}=\right.$ 40-43.7 $\left.\mathrm{erg} \mathrm{s}^{-1}\right)$ and high $\left(\log L_{14-195}=43.7-46 \mathrm{erg} \mathrm{s}^{-1}\right)$ luminosities, respectively; the green diamond is the measurement from Burlon et al. (2011) on an earlier version of the Swift-BAT hard band catalogue, with no luminosity cut. The light grey shaded area is from Buchner et al. (2015) for $\log L_{X}=43.2-43.6 \mathrm{erg} \mathrm{s}^{-1}$. All these measurements point towards an increase of $f_{\mathrm{CT}}$ from low to high redshift (with the exception of the Buchner et al. results).

Performing a linear fit between $f_{\mathrm{CT}}$ and $z$ to our data points at high luminosity, we get a slope of $m \sim 0.13$. For an easier comparison with literature results we compute the dependency adopting an expression such as $f_{\mathrm{CT}}=\beta(1+z)^{\alpha}$. The best-fitting parameters are $\beta=0.11_{-0.04}^{+0.05}$ and $\alpha=1.11_{-0.12}^{+0.14}$. Interestingly, this relation perfectly fits also the data point at high luminosity from Ricci et al.

\footnotetext{
${ }^{5}$ We recall, however, that Marchesi et al. (2018) showed that these low- $z$ results may be overestimated by $\sim 20$ per cent based on NUSTAR data. But see also La Caria et al. (2018) for a smaller discrepancy in the other direction between pre- and post-NUSTAR data.
}

(2015, cyan square in Fig. 8, right). The slope of $f_{\mathrm{CT}}$ as a function of redshift at high luminosities is therefore significantly steeper than the slope found in BU12 with similar methods for lower luminosities $(\alpha \sim 0.3)$, suggesting a faster evolution of $f_{\mathrm{CT}}$ for more luminous systems. For comparison the evolution of the obscured fraction (again at low luminosities), measured in Liu et al. (2017), is fitted with best-fitting parameters $\beta=0.42 \pm 0.09$ and $\alpha=$ $0.60 \pm 0.17$.

\section{MORPHOLOGY OF CT AGN}

We collected all the available HST-ACS I-band images from the COSMOS mosaic (Koekemoer et al. 2007, I-band magnitude limit 27.2) for the 67 CT AGN candidates. We adopted a simplified visual classification scheme comprising, on one side, isolated/undisturbed morphologies, and, on the other side, sources clearly in merger state or with post-merger features such as tidal tails and disturbed morphologies or with close companions. ${ }^{6} 17$ sources have no classification since the HST counterpart is absent or too faint to determine any morphological feature $(I>25), 16$ out of these 17 have $z>$ 1.5 .

Our morphological classification is supported by the comparison with the Tasca Morphology Catalogue (Tasca et al. 2009). All the 50 CT AGN with visual classification have a counterpart in the catalogue, and 90 per cent of the sources classified by us as merging or post-merging systems (panels b and c of Fig. 9, respectively) are classified as irregulars in at least two of the three classification schemes of that catalogue, based on different parametric estimates.

$L_{\mathrm{Bol}}$ for all the sources has been derived using the Marconi et al. (2004) X-ray bolometric correction. The final sample of 50 sources with morphological information has been divided in $L_{\mathrm{Bol}}$ bins of 1 dex width. The bin $\log \left(L_{\mathrm{Bol}}\right)=44-45 \mathrm{erg} \mathrm{s}^{-1}$ comprises only three sources and is therefore ignored. As in the previous sections, each of the CT AGN in our sample has been weighted by the fraction of its $N_{\mathrm{H}} \mathrm{PDF}$ above $10^{24} \mathrm{~cm}^{-2}$, and this number has been corrected for classification bias and survey sensitivity. The errors are computed adopting the Bayesian approach of Cameron (2011).

We compared our results in Fig. 10 with results from several previous studies, and from the Hickox et al. (2014) model. The literature results at intermediate luminosities $\left(\log L_{\mathrm{Bol}}=45-46 \mathrm{erg} \mathrm{s}^{-1}\right.$; Kocevski et al. 2012; Lanzuisi et al. 2015; Del Moro et al. 2016) give a merger fraction both for the CT candidate samples (magenta) and

\footnotetext{
${ }^{6}$ In five cases the CT AGN has a companion within 3 arcsec that is bright enough to have a photometric redshift in the COSMOS2015 catalogue (Laigle et al. 2016), and we checked that in all cases the two sources have comparable redshifts.
} 

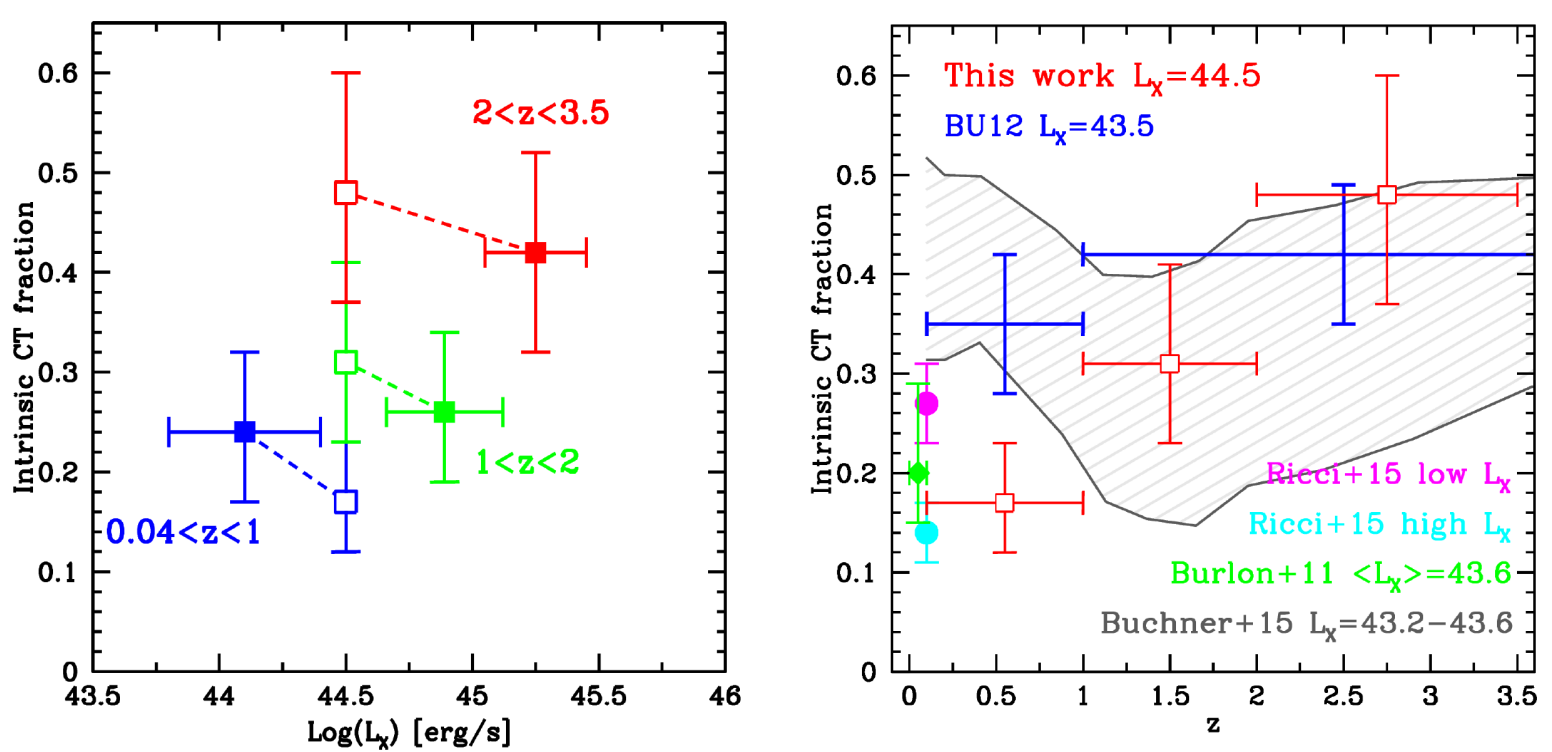

Figure 8. Left: intrinsic fraction of CT AGN as a function of $L_{\mathrm{X}}$, observed (filled squares) and extrapolated to $\log \left(L_{\mathrm{X}}\right)=44.5 \mathrm{erg} \mathrm{s}{ }^{-1}$ (empty squares) for the three redshift bins: blue for z1, green for z2, and red for z3. Right: intrinsic fraction of CT AGN as a function of redshift for $\log \left(L_{\mathrm{X}}\right)=44.5 \mathrm{erg} \mathrm{s}{ }^{-1}$ (red empty squares). Data points from the literature are from Burlon et al. (2011 green diamond), Ricci et al. (2015 cyan and magenta circles), and BU12 (blue crosses). The light grey dashed area is from Buchner et al. (2015).
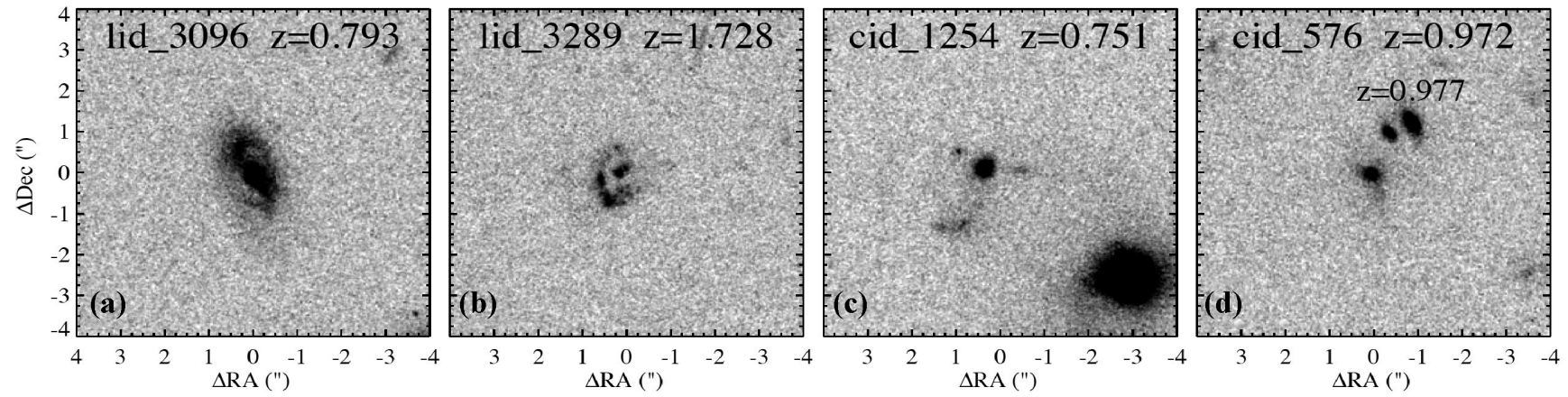

Figure 9. HST/ACS I-band cut-outs (Koekemoer et al. 2007) of four CT AGN candidates. They show examples of an isolated spiral (barred) galaxy (a), a merging system (b), a galaxy with tidal tails (c), and a group of close companions with similar redshifts (d). The cut-outs have a size of $8 \times 8$ arcsec ${ }^{2}$.

for the C-thin parent samples (blue) at the same luminosity, while for very high luminosities (black points) morphological results refer to $z \gtrsim 2$ dust-reddened quasars (Glikman et al. 2015; Fan et al. 2016), not necessarily CT. The results from our large sample of CT AGN (red points) allow to confirm that highly obscured AGN at high luminosities show an increase in merger fraction with respect to the C-thin parent sample. Our results are also in agreement with the AGN-galaxy co-evolution model of Hickox et al. (2014), in which galaxy mergers play a prominent role in triggering the most luminous and obscured AGN at $z \sim 2$. We note that the average redshift of the bins $\log \left(L_{\mathrm{Bol}}\right)=45-46,46-47$, and $47-48 \mathrm{erg} \mathrm{s}^{-1}$ are $z=0.93,1.83$, and 2.38 , respectively. Also the data points from the literature at the highest luminosities (from Glikman et al. 2015; Fan et al. 2016) are derived for sources at $z>2$. Therefore with these data sets it is not possible to disentangle a luminosity from a redshift dependence of the merger fraction.

\section{CONCLUSIONS}

We compiled one of the largest samples of CT AGN candidates at high redshift (67 individual sources) from the COSMOS Legacy point source catalogue. For comparison, there are $100 \mathrm{CT}$ AGN candidates in Brightman et al. (2014) summing three Chandra surveys and 165 CT AGN candidates in Buchner et al. (2015) from four different surveys. Our sample was selected applying a physically motivated model for the X-ray emission, and MCMC methods to efficiently explore the parameter space $N_{\mathrm{H}}$ versus $L_{\mathrm{X}}$ and taking into account the full PDF of these quantities in our analysis. The number of 'effective' CT AGN derived from the sum of the $N_{\mathrm{H}}$ PDF above $10^{24} \mathrm{~cm}^{-2}$ is $38.5,41.9$ after correction for identification bias. The two main results of this work are as follows.

(i) The fraction of CT AGN increases as a function of redshift from $\sim 0.2$ at $z=0.1-1$ to $\sim 0.3$ at $z=1-2$, and to $\sim 0.5$ at $z=$ $2-3.5$. These values are derived from the observed fraction of CT AGN in each redshift bin, rescaled to a common luminosity range of $\log \left(L_{\mathrm{X}}\right)=44.5 \mathrm{erg} \mathrm{s}^{-1}$. This evolution can be parametrized as $f_{\mathrm{CT}}=$ $0.11(1+z)^{1.11}$. A similar trend, with values of $0.30,0.45$, and 0.55 , was found for the global CT fraction by a qualitative comparison of the $\log N-\log S$ of the CT AGN in the same redshift bins, with the CXB model of Akylas et al. (2012).

(ii) The fraction of CT AGN in merging/interacting systems is systematically higher, by a factor $2.5-3$, than that observed in the 


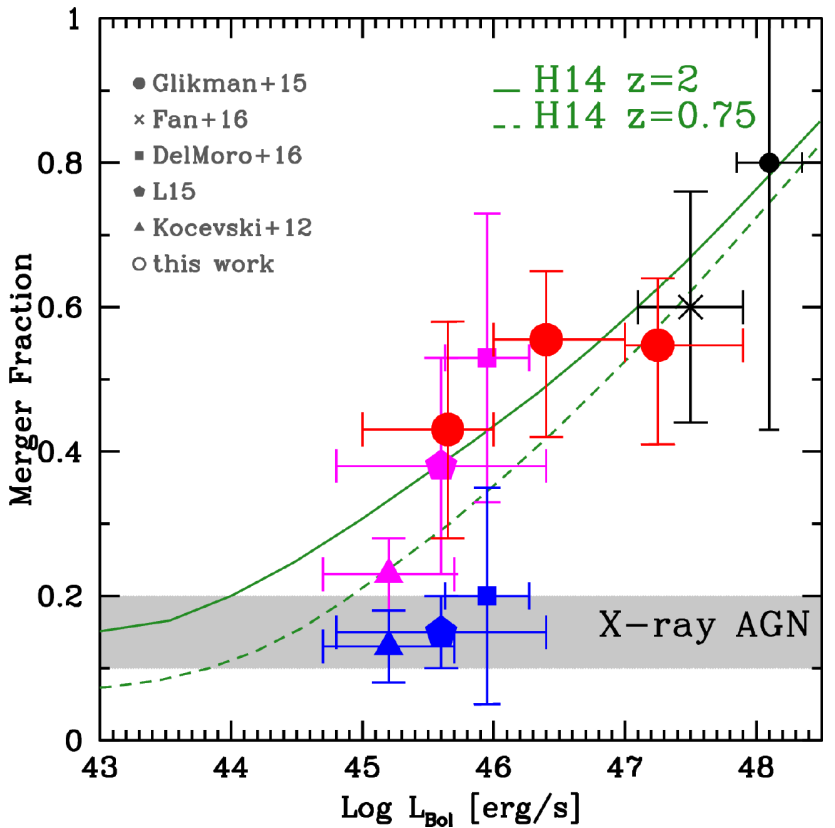

Figure 10. Fraction of merger/disturbed morphology for several samples of $\mathrm{CT}$ and non-CT AGN at different $L_{\mathrm{Bol}}$. The red circles show the measurements for our sample, divided in three $L_{\mathrm{Bol}}$ bins. The magenta points show fractions for different CT AGN samples in the literature. Blue points are the parent sample of non-CT AGN in each of these studies. The black points at high luminosities are merger fractions for dust-reddened quasars (Glikman et al. 2015; Fan et al. 2016) at $z \gtrsim 2$. The green thick (dashed) line shows the prediction of the Hickox et al. (2014) model of AGN-galaxy co-evolution at $z=2(z=0.75)$.

parent sample of C-thin AGN in several other studies. This increase also has a positive dependence on the $L_{\mathrm{Bol}}$. Given the redshift and luminosity distribution of the samples, it is not possible to disentangle a luminosity from a redshift dependence of the merger fraction.

Our interpretation of these results, together with other pieces of evidences in the literature (see Alexander \& Hickox 2012; Netzer 2015 for recent reviews), is that at high redshift, the simplest version of the 'unification scheme' for AGN (Antonucci 1993) does not hold anymore, and the orientation with respect to a nuclear obscuring torus is not the main driver of the differences between obscured and unobscured AGN. Instead, the conditions of the AGN host galaxy, i.e. amount and distribution of cold gas, nuclear star formation, level of interaction with neighbours, etc., may play a major role in determining the amount of obscuration we measure in the X-ray.

Such 'large-scale' obscuration should exist in addition to the classical nuclear dusty region commonly referred to as torus (see e.g. Elvis 2012). The relative importance of these different components will depend on the host galaxy environment, gas and dust properties that at high redshift may favour the presence of large amount of obscuring material (e.g. an augmented gas fraction; see e.g. Carilli \& Walter 2013; Scoville et al. 2017; Darvish et al. 2018).

Some recent results from AGN-host population studies also point in this direction, deriving a positive correlation between obscuration, at least in the $\mathrm{C}$-thin regime, and host gas mass or stellar mass ( Rodighiero et al. 2015; Lanzuisi et al. 2017). As an extreme example for the CT regime, Gilli et al. (2014) showed that the CT absorber observed in an obscured quasi-stellar object (QSO) hosted in an ultraluminous IR galaxy at $z=4.75$ can be fully accounted for by the amount of gas and its compactness measured by Atacama Large Millimeter/submillimeter Array (ALMA).

Indeed the gas fraction increases from $z=0$ to 3 derived from ALMA dust continuum observations (Scoville et al. 2014, 2017; Darvish et al. 2018) goes in this direction. We envisage that direct cold gas mass and size measurement through ALMA CO observations in CT AGN hosts at high redshift will help to shed light on the nature of the nuclear absorber and possibly its connection with the host star formation properties (e.g. Perna et al. 2018).

While the Chandra deep fields such as CDF-S, CDF-N, and COSMOS represent the current limit for the search of CT AGN at high redshift, large samples (hundreds) of such sources will be detected and routinely identified (Georgakakis et al. 2013) in the planned extragalactic survey (Aird et al. 2013) of the Wide Field Imager (WFI) onboard the next generation ESA X-ray mission Athena (Nandra et al. 2013).

As an example, the current Athena-WFI design will allow to collect, in the medium ( $600 \mathrm{ks}$ exposure) tier of the survey, $\sim 10^{4}$ full band net counts for a source like lid_283. This is the highest redshift CT in our sample at $z=3.465$ and $\log \left(F_{2-10}\right)=-14 \mathrm{erg} \mathrm{cm}^{-2} \mathrm{~s}^{-1}$. Such spectral quality will result in a $N_{\mathrm{H}}$ uncertainty of $<5$ per cent. Around 3500 full band net counts will be collected for a source with similar intrinsic luminosity moved at $z=6$, with a derived $N_{\mathrm{H}}$ uncertainty of $\sim 10$ per cent.

The NASA proposed Lynx mission (Gaskin et al. 2015) is planned to have a 0.5 arcsec point spread function (PSF), and the deep surveys performed with its High Definition X-ray Imager will be able to resolve high- $z$ AGN where Athena might be affected by source confusion, making possible to extend the constraints for the CT fraction at even fainter luminosities and higher redshifts.

\section{ACKNOWLEDGEMENTS}

The authors thank the referee for the constructive comments and recommendations that helped to improve the readability and quality of the paper. This research has made use of data obtained from the Chandra Data Archive and software provided by the Chandra Xray Center (CXC) in the CIAO application package. The authors acknowledge financial support from the CIG grant 'eEASY' no. 321913, from ASI-INAF grant no. 2014-045-R.0, and from PRININAF-2014 ('Windy Black Holes Combing Galaxy Evolution').

\section{REFERENCES}

Aird J. et al., 2013, preprint (arXiv:1306.2325)

Akylas A., Georgakakis A., Georgantopoulos I., Brightman M., Nandra K., 2012, A\&A, 546, A98

Akylas A., Georgantopoulos I., Ranalli P., Gkiokas E., Corral A., Lanzuisi G., 2016, A\&A, 594, A73

Alexander D., Chary R. R., Pope A., et al., 2008, ApJ, 687, 835

Alexander D. M., Hickox R. C., 2012, New Astron. Rev., 56, 93

Antonucci R., 1993, ARA\&A, 31, 473

Arnaud K. A., 1996, in Jacoby G. H., Barnes J., eds, ASP Conf. Ser. Vol. 101, Astronomical Data Analysis Software and Systems V. Astron. Soc. Pac., San Francisco, p. 17

Asmus D., Gandhi P., Honig S. F., et al., 2015, MNRAS, 454, 766

Ballantyne D. R., Draper A. R., Madsen K. K., Rigby J. R., Treister E., 2011, ApJ, 736, 56

Bianchi S., Guainazzi M., Matt G., et al., 2009, A\&A, 495, 421

Brightman M., Nandra K., Salvato M., Hsu L.-T., Rangel C., 2014, MNRAS, 443, 1999

Brightman M., Ueda Y., 2012, MNRAS, 423, 702 ( BU12)

Brunner H., Cappelluti N., Hasinger G., et al., 2008, A\&A, 479, 283 
Brusa M. et al., 2010, ApJ, 716, 348

Buchner J. et al., 2014, A\&A, 564, A125

Buchner J. et al., 2015, ApJ, 802, 89

Burlon D., Ajello M., Greiner J., Comastri A., Merloni A., Gehrels N., 2011, ApJ, 728, 58

Cameron E., 2011, Publ. Astron. Soc. Aust., 28, 128

Cappelluti N. et al., 2009, A\&A, 497, 635

Carilli C. L., Walter F., 2013, ARA\&A, 51, 105

Cash W., 1979, ApJ, 228, 939

Castelló-Mor N. et al., 2013, A\&A, 556, A114

Civano F. et al., 2012, ApJS, 201, 30

Civano F. et al., 2015, ApJ, 808, 185

Civano F. et al., 2016, ApJ, 819, 62

Comastri A., Setti G., Zamorani G., Hasinger G., 1995, A\&A, 296, 1

Comastri A. et al., 2011, A\&A, 526, L9

Darvish B., Scoville N. Z., Martin C., et al., 2018, ApJ, 860, 11

Del Moro A. et al., 2016, MNRAS, 456, 2105

Delvecchio I. et al., 2015, MNRAS, 449, 373

Elvis M., 2012, J. Phys. Conf. Ser., 372, 012032

Elvis M. et al., 2009, ApJS, 184, 158

Fan L. et al., 2016, ApJ, 822, L32

Gandhi P., Horst H., Smette A., et al., 2009, A\&A, 502, 457

Gaskin J. A. et al., 2015, Proc. SPIE, 9601, 96010J

Georgakakis A. et al., 2013, preprint (arXiv:1306.2328)

Georgantopoulos I. et al., 2011, A\&A, 534, A23

Georgantopoulos I. et al., 2013, A\&A, 555, A43

George I. M., Fabian A. C., 1991, MNRAS, 249, 352

Gilli R., Comastri A., Hasinger G., 2007, A\&A, 463, 79 ( G07)

Gilli R. et al., 2014, A\&A, 562, A67

Glikman E., Simmons B., Mailly M., Schawinski K., Urry C. M., Lacy M., 2015, ApJ, 806, 218

Goodman J., Weare J., 2010, Commun. Appl. Math. Comput. Sci., 5, 65

Goulding A. D., Alexander D. M., Mullaney J. R., et al., 2011, MNRAS, 411,1231

Hasinger G., 2008, A\&A, 490, 905

Hasinger G. et al., 2007, ApJS, 172, 29

Hickox R. C., Mullaney J. R., Alexander D. M., Chen C.-T. J., Civano F. M., Goulding A. D., Hainline K. N., 2014, ApJ, 782, 9

Ilbert O. et al., 2009, ApJ, 690, 1236

Kocevski D. D. et al., 2012, ApJ, 744, 148

Koekemoer A. M. et al., 2007, ApJS, 172, 196

Laigle C. et al., 2016, ApJS, 224, 24

Lansbury G. B. et al., 2017, ApJ, 846, 20

Lanzuisi G., Delvecchio I., Berta S., et al., 2017, A\&A, 602A, 123

Lanzuisi G., Piconcelli E., Fiore F., Feruglio C., Vignali C., Salvato M., Gruppioni C., 2009, A\&A, 498, 67

Lanzuisi G. et al., 2013, MNRAS, 431, 978

Lanzuisi G. et al., 2015, A\&A, 573, A137

Liu T. et al., 2017, ApJS, 232, 8

Marchesi S., Ajello M., Marcotulli L., Comastri A., Lanzuisi G., Vignali C., 2018, ApJ, 854, 49

Marchesi S. et al., 2016a, ApJ, 817, 34

Marchesi S. et al., 2016b, ApJ, 830, 100 ( M16)

Marconi A., Risaliti G., Gilli R., Hunt L. K., Maiolino R., Salvati M., 2004, MNRAS, 351, 169

Masini A. et al., 2018, ApJS, 235, 17

Murphy K. D., Yaqoob T., 2009, MNRAS, 397, 1549 ( MY09)

Nandra K. et al., 2013, preprint (arXiv:1306.2307)

Netzer H., 2015, ARA\&A, 53, 365

Noguchi K., Terashima Y., Ishino Y., Hashimoto Y., Koss M., Ueda Y., Awaki H., 2010, ApJ, 711, 144

Perna M., Sargent M. T., Brusa M., et al., 2018, preprint (arXiv:1807.03378)

Piconcelli E., Jimenez-Bailón E., Guainazzi M., Schartel N., Rodríguez-

Pascual P. M., Santos-Lleó M., 2005, A\&A, 432, 15

Ricci C., Ueda Y., Koss M. J., Trakhtenbrot B., Bauer F. E., Gandhi P., 2015, ApJ, 815, L13

Risaliti G., Maiolino R., Salvati M., 1999, ApJ, 522, 157

Rodighiero S., Brusa M., Daddi E., et al., 2015, ApJ, 800L, 10
Salvato M. et al., 2011, ApJ, 742, 61

Scoville N. et al., 2007, ApJS, 172, 1

Scoville N. et al., 2014, ApJ, 783, 84

Scoville N. et al., 2017, ApJ, 837, 150

Stern D., 2015, ApJ, 807, 129

Suh H. et al., 2017, ApJ, 841, 102

Tasca L. A. M. et al., 2009, A\&A, 503, 379

Tozzi P. et al., 2006, A\&A, 451, 457

Treister E., Urry C. M., Virani S., 2009, ApJ, 696, 110

Ueda Y., Akiyama M., Hasinger G., Miyaji T., Watson M. G., 2014, ApJ, 786, 104

Vito F., Gilli R., Vignali C., et al., 2014, MNRAS, 445, 3557

Wachter K., Leach R., Kellogg E., 1979, ApJ, 230, 274

Zappacosta L. et al., 2018, ApJ, 854, 33

\section{SUPPORTING INFORMATION}

Supplementary data are available at MNRAS online.

Figure D1. Unfolded spectrum and NH PDF for the CT candidates. In green the obscured power law, in blue the reflection component, in magenta the emission lines component, and in orange the scattered power law.

Please note: Oxford University Press is not responsible for the content or functionality of any supporting materials supplied by the authors. Any queries (other than missing material) should be directed to the corresponding author for the article.

\section{APPENDIX A: PARAMETER SPACE EXPLORATION}

We adopted an MCMC approach to explore the parameter space in the spectral fit. Once the best fit with the standard C-stat likelihood is obtained, we run the MCMC code implemented in XSPEC (v. 12.9.1). We use the Goodman-Weare algorithm (Goodman \& Weare 2010) with $10^{4}$ steps and $10^{3}$ burn in steps to ensure convergence and efficiently explore the parameter space. The marginalization over the parameter of interest gives the full PDF distribution. The errors reported in Table 1 are obtained by the classical command 'error'

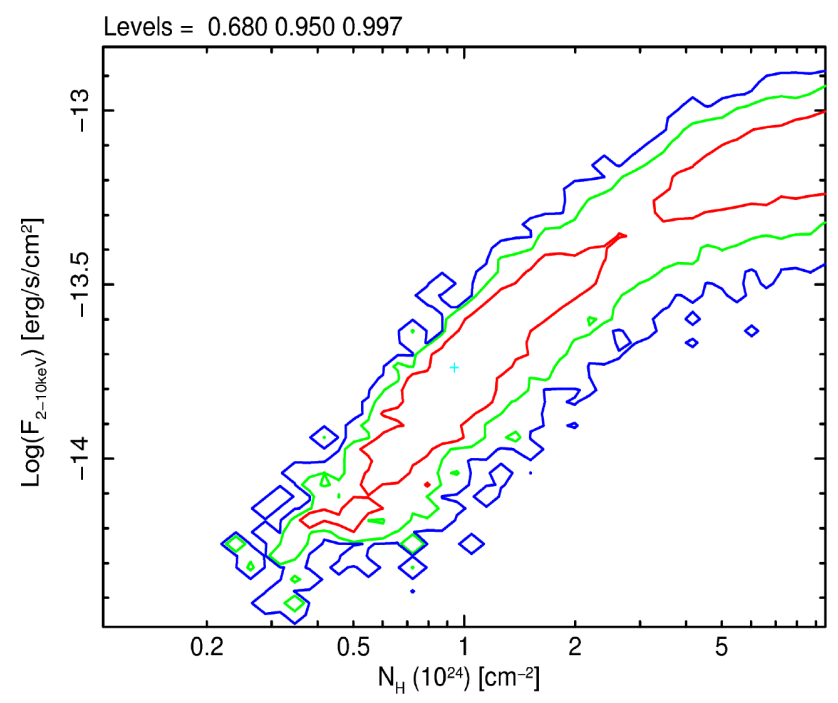

Figure A1. PDF for two parameters of interest, $N_{\mathrm{H}}$ and intrinsic flux, for source lid_3516. In this case there are two distinct minima of the fit statistic. The MCMC method is able to properly take into account both of them. 

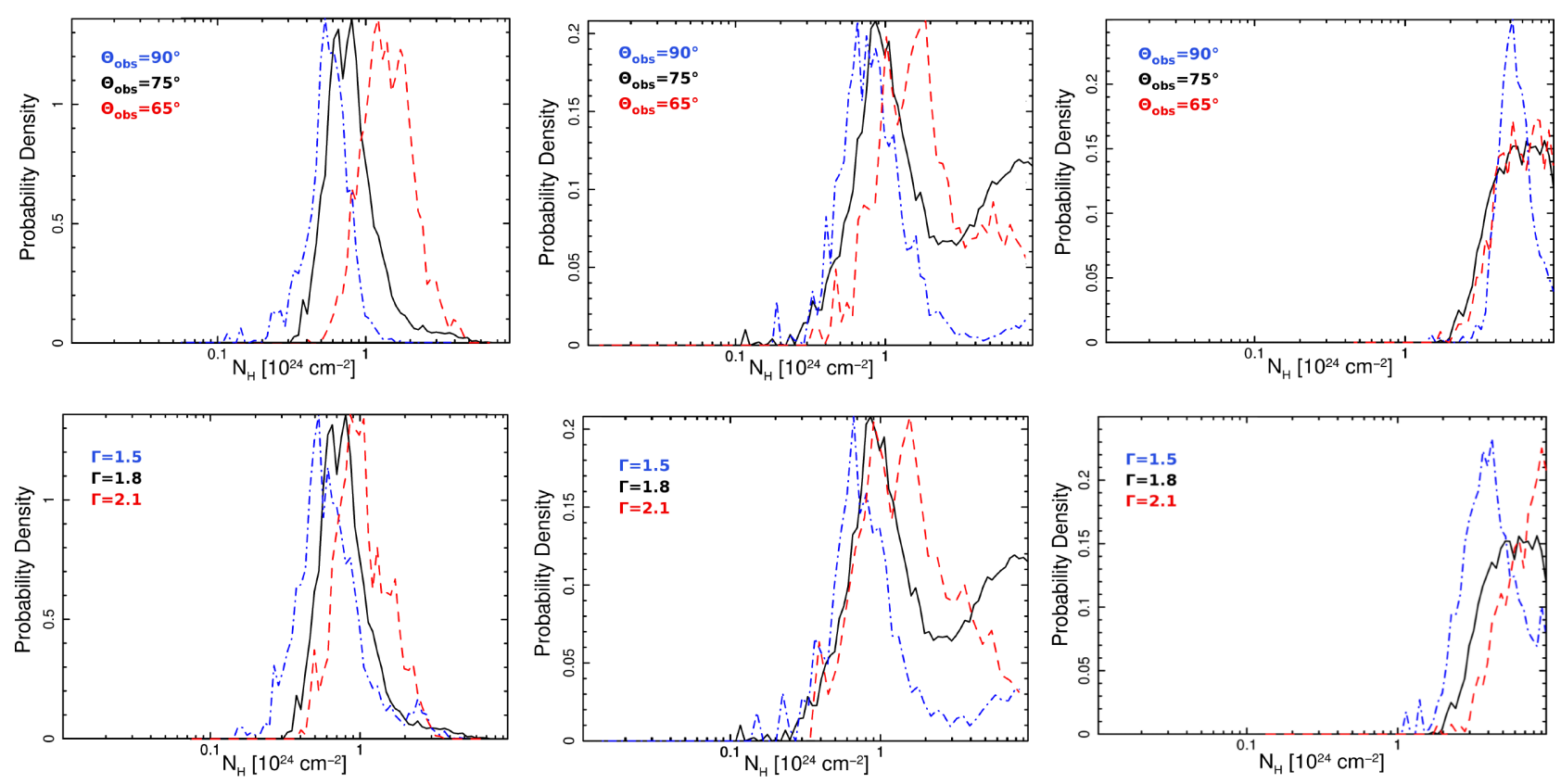

Figure B1. Top: $N_{\mathrm{H}}$ PDF for different values of $\Theta_{\mathrm{obs}}: 65^{\circ}, 75^{\circ}$, and $90^{\circ}$ (red, black, and blue, respectively) for the sources shown in Fig. 2 (lid_633, lid_3516, and lid_390). Bottom: $N_{\mathrm{H}}$ PDF for different values of $\Gamma: 2.1,1.8$, and 1.5 (red, black, and blue, respectively).

in XSPEC, but in this case the program uses the chain to derive the confidence interval at a given c.l. directly from the PDF.

Fig. A1 shows an example of a double-peaked PDF in the parameter space $N_{\mathrm{H}}$ versus intrinsic flux, for source lid_3516. The red, green, and blue contours show the $1 \sigma, 2 \sigma$, and $3 \sigma$ c.l., respectively. The standard methods for error estimation would fail to correctly estimate the uncertainties in these cases. Such a probability distribution in $N_{\mathrm{H}}$ is rather common in X-ray spectra of CT candidates that often allow for two solutions, one with $N_{\mathrm{H}}$ below the CT threshold and lower $L_{\mathrm{X}}$, and a second at higher $N_{\mathrm{H}}$ and intrinsic luminosity (see also Buchner et al. 2014).

Finally, we computed the fraction of PDF that each source shows above the CT threshold, and then apply a probabilistic approach when analysing the number counts of samples of CT AGN: the effective number of $\mathrm{CT}$ is the sum of the fraction of the PDF above $\mathrm{CT}$ values for each source in the sample.

\section{APPENDIX B: IMPACT OF DIFFERENT ASSUMED PARAMETERS}

Here we show the impact of the use of different values of the fixed parameters in the model for CT AGN, such as $\Gamma$ and inclination angle $\Theta_{\text {obs }}$, on the determination of $N_{\mathrm{H}}$. The top panels of Fig. B1 show the variation of the $N_{\mathrm{H}}$ PDF for different assumed torus inclination angles: $\Theta_{\mathrm{obs}}=65^{\circ}, 75^{\circ}$, and $90^{\circ}$ (red, black, and blue, respectively) for the three sources shown in Fig. 2 as representative of the sample. The lower panels show the variation for different assumed photon indices: $\Gamma=2.1,1.8$, and 1.5 (red, black, and blue, respectively) for the same sources.

The change in $\Gamma$ has the effect of shifting the $N_{\mathrm{H}}$ PDF by $\sim 0.1$ dex in both directions. The $\Gamma$ range explored corresponds to $\sim 1.5 \sigma$ of the observed distribution (e.g. Piconcelli et al. 2005; Bianchi et al. 2009).

The change in $\Theta_{\text {obs }}$ has a slightly larger effect, shifting the $N_{\mathrm{H}}$ PDF by $\sim 0.1$ dex for $90^{\circ}$ and by $\sim 0.3$ dex for $65^{\circ}$. This is due to the fact that, in the geometry of MYTORUs model, the line of sight intercepts the torus only for $\Theta_{\text {obs }}>60^{\circ}$, and at $65^{\circ}$ the section of the torus intercepted is very small. Therefore the best-fitting $N_{\mathrm{H}}$ (defined as the equatorial $N_{\mathrm{H}}$; see MY09) needs to be higher to reproduce a given spectral shape, with respect to larger $\Theta_{\text {obs }}$. We note also that for heavily CT sources $\left(F_{\mathrm{PDF}}^{\mathrm{CT}}=1\right)$ the $N_{\mathrm{H}} \mathrm{PDF}$ is less affected by these changes.

\section{APPENDIX C: BIAS CORRECTIONS}

\section{C1 Differential sky coverage}

The sky coverage of COSMOS Legacy was computed converting count rates into fluxes in different bands, assuming a power-law spectrum with $\Gamma=1.4$ and Galactic $N_{\mathrm{H}}=2.6 \times 10^{20} \mathrm{~cm}^{-2}$ (Civano et al. 2016). This is appropriate if averaging over the intrinsic $N_{\mathrm{H}}$ of the whole AGN population, and correctly applies, on average, also to the M16 sample of sources with more than 30 net counts in the 0.5-7 keV band.

However, to correctly derive $N_{\mathrm{H}}$ distributions we need to take into account the fact that the conversion between detected counts and emitted flux depends on the spectral shape (see e.g. Vito et al. 2014; Liu et al. 2017). In the case of our sample of CT AGN, the source $N_{\mathrm{H}}$ and redshift change the conversion factor between counts and flux in the sense that 30 full band counts for a CT source typically correspond to a higher observed $2-10 \mathrm{keV}$ flux with respect to an average power law.

We therefore recomputed the $2-10 \mathrm{keV}$ sky coverage in the three redshift bins adopted in the $\log N-\log S$ analysis for a spectrum with $N_{\mathrm{H}}=10^{24} \mathrm{~cm}^{-2}$ (see Fig. C1), and applied these corrected sky coverages when deriving the source counts for the $\log N-\log S$. The differences in sky coverage, i.e. the difference of area at a given flux, have a maximum of 30,10, and 3 per cent for $\mathrm{z} 1, \mathrm{z} 2$, and $\mathrm{z} 3$, respectively, for the faintest fluxes covered by our sample. The effect of these differences is however very small, appreciably increasing the cumulative number counts of CT AGN only in the last two data points of Fig. 5 and only in the first redshift bin. This correction 


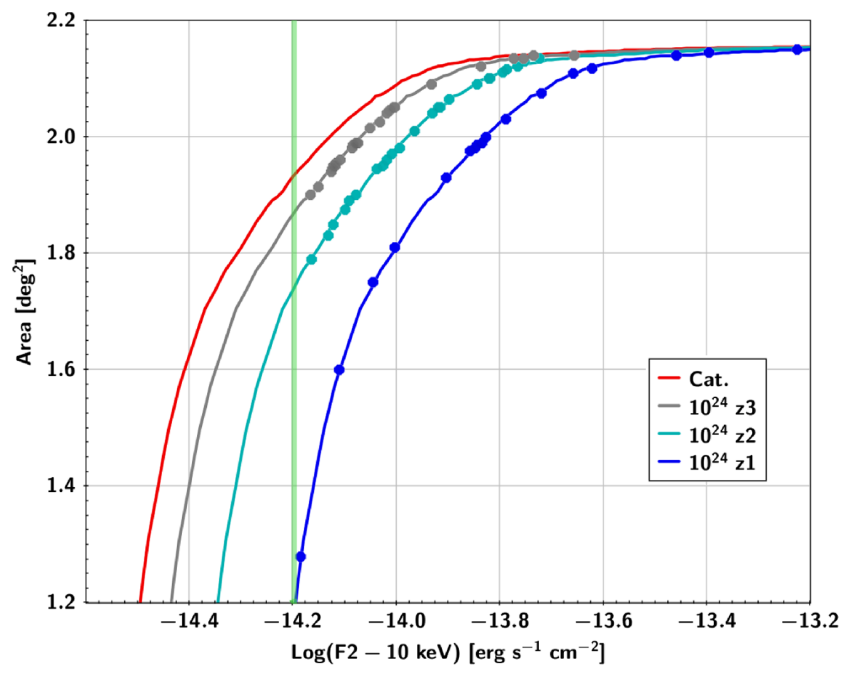

Figure C1. 2-10 keV sky coverage of the COSMOS Legacy survey, as computed for the whole catalogue (red curve) and as recomputed here for sources with $N_{\mathrm{H}}=10^{24} \mathrm{~cm}^{-2}$, in the three redshift bins (blue z1, cyan z2, and grey z3). The green vertical line shows the nominal flux limit for the CT sample.

instead does not affect the conclusions of Section 5 (Fig. 8) since, as explained in Section 5, we are taking into account only sources well above the survey flux limit.

\section{C2 Classification bias}

We performed simulations to derive the fraction of CT sources correctly identified as such for a given flux and redshift. We followed
BU12 and simulated $10^{3}$ spectra in each of the three redshift bins and five intervals of flux, assuming $N_{\mathrm{H}}=10^{24} \mathrm{~cm}^{-2}$ and $\Gamma=1.8$, and a secondary power law with $f_{\text {scatt. }}=3$ per cent fixed. We then performed the same spectral analysis described in Section 2.2 and analysed the derived PDF distributions. Since the input $N_{\mathrm{H}}$ for the simulation is centred at $10^{24} \mathrm{~cm}^{-2}$, we expect the fraction of PDF above this value to be $\sim 0.5$ (the PDF should be symmetric around the input value). Indeed, this value is recovered for the highest flux bins, while at the faintest fluxes (below $10^{-14} \mathrm{erg} \mathrm{cm}^{-2} \mathrm{~s}^{-1}$ ) the average PDF fraction above $10^{24} \mathrm{~cm}^{-2}$ is significantly lower than the expected 0.5 . This translates into a fraction of correctly identified CT sources of $\sim 0.6,0.8$, and 0.95 at $\mathrm{z} 1, \mathrm{z} 2$, and $\mathrm{z} 3$, respectively (i.e. it is easier to identify a faint CT source if it is located at high redshift than if it is at low $z$ ). We note that sources with $f_{\mathrm{CT}}$ close to one can have this value $>1$ once the classification bias correction is applied. This has the meaning of accounting for other sources with similar $N_{\mathrm{H}}$ PDF and number of counts that are instead missing due to the classification bias. We also note that the choice of limiting the analysis to sources with more than 30 counts translates into a less severe correction for misclassification, with respect to BU12, that has no cut in number of counts.

Finally, we simulated $10^{3}$ spectra for $\log \left(N_{\mathrm{H}}\right)=21,22$, and $23\left(\mathrm{~cm}^{-2}\right)$ in the three redshift bins and five flux intervals. The contamination, i.e. C-thin sources with a sizable fraction of the $N_{\mathrm{H}}$ $\mathrm{PDF}$ in the CT regime, is negligible at these flux and counts levels: the average $N_{\mathrm{H}}$ PDF of the simulated spectra with input $\log \left(N_{\mathrm{H}}\right)=$ $23\left(\mathrm{~cm}^{-2}\right)$ at $\mathrm{z} 1$ and the lowest fluxes (below $10^{-14} \mathrm{erg} \mathrm{cm}^{-2} \mathrm{~s}^{-1}$ ) has only a fraction of 0.02 above the $\mathrm{CT}$ threshold, while the others have none.

This paper has been typeset from a $\mathrm{T}_{\mathrm{E}} \mathrm{X} / \mathrm{L} \mathrm{T} \mathrm{E}$ file prepared by the author. 